\title{
Dyadic BMO on the bidisk
}

\section{Óscar Blasco and Sandra Pott}

\begin{abstract}
We give several new characterizations of the dual of the dyadic Hardy space $H^{1, d}\left(\mathbb{T}^{2}\right)$, the so-called dyadic BMO space in two variables and denoted $\mathrm{BMO}_{\text {prod. }}^{d}$. These include characterizations in terms of Haar multipliers, in terms of the "symmetrised paraproduct" $\Lambda_{b}$, in terms of the rectangular BMO norms of the iterated "sweeps", and in terms of nested commutators with dyadic martingale transforms. We further explore the connection between $\mathrm{BMO}_{\text {prod }}^{d}$ and John-Nirenberg type inequalities, and study a scale of rectangular BMO spaces.
\end{abstract}

\section{Introduction}

Throughout the paper $\mathcal{D}$ denotes the set of dyadic intervals in the unit circle $\mathbb{T}$. In the case of the bicircle $\mathbb{T}^{2}, \mathcal{D}_{1}$ denotes the dyadic intervals in the first, $\mathcal{D}_{2}$ the dyadic intervals in the second variable. We write $\mathcal{R}=\mathcal{D}_{1} \times \mathcal{D}_{2}$ for the dyadic rectangles, $|I|$ for the length of $I \in \mathcal{D}$ and $|R|$ for the area of $R \in \mathcal{R},\left(h_{I}\right)_{I \in \mathcal{D}}$ stands for the Haar basis in $L^{2}(\mathbb{T})$ and $\left(h_{R}\right)_{R \in \mathcal{R}}$ for the product Haar basis of $L^{2}\left(\mathbb{T}^{2}\right)$.

Here

$$
h_{I}(t)=\frac{1}{|I|^{1 / 2}}\left(\chi_{I^{+}}(t)-\chi_{I^{-}}(t)\right)
$$

for each dyadic interval $I \in \mathcal{D}$, where $I^{-}$denotes the left half of $I$, and $I^{+}$ denotes the right half of $I$. For each dyadic rectangle $R=I \times J \in \mathcal{R}, h_{R}$ is defined by

$$
h_{R}(s, t)=h_{I}(s) h_{J}(t) .
$$

We denote by $\mathcal{H}_{00}$ the space of all functions in $L^{2}\left(\mathbb{T}^{2}\right)$ which have a finite expansion in the product Haar basis.

2000 Mathematics Subject Classification: 42B30, $47 \mathrm{~B} 35$.

Keywords: BMO on the bidisk, Carleson measures, Haar multipliers. 
Given $g \in L^{2}(\mathbb{T})$, we use the notation $g_{I}=\left\langle g, h_{I}\right\rangle$ and $m_{I} g=\frac{1}{|I|} \int_{I} g(t) d t$. Similarly, given $f \in L^{2}\left(\mathbb{T}^{2}\right)$, we use the notation $f_{R}=\left\langle f, h_{R}\right\rangle, f_{I}(s)=$ $\left\langle f(\cdot, s), h_{I}\right\rangle, m_{I} f(s)=\frac{1}{|I|} \int_{I} f(t, s) d t, f_{J}(t)=\left\langle f(t, \cdot), h_{J}\right\rangle$ and $m_{J} f(t)=$ $\frac{1}{|J|} \int_{J} f(t, s) d s$. Therefore

$$
f(t, s)=\sum_{R \in \mathcal{R}} f_{R} h_{R}(t, s)=\sum_{I \in \mathcal{D}} f_{I}(s) h_{I}(t)=\sum_{J \in \mathcal{D}} f_{J}(t) h_{J}(s) .
$$

Let $P_{I} g=\left(g-m_{I} g\right) \chi_{I}$ for $g \in L^{2}(\mathbb{T})$. Observe that $P_{I}$ is the orthogonal projection on the subspace spanned by the Haar functions $h_{I^{\prime}}, I^{\prime} \in \mathcal{D}, I^{\prime} \subseteq I$. If $g=\sum_{I \in \mathcal{D}} g_{I} h_{I}$, then

$$
P_{I} g=\sum_{I^{\prime} \in \mathcal{D}, I^{\prime} \subseteq I} h_{I^{\prime}} g_{I^{\prime}}
$$

Similarly, for each measurable set $\Omega \subseteq \mathbb{T}^{2}$, let $P_{\Omega}$ be the orthogonal projection on the subspace spanned by the Haar functions $h_{R^{\prime}}, R^{\prime} \in \mathcal{R}$, $R^{\prime} \subseteq \Omega$. In particular, for each dyadic rectangle $R=I \times J \in \mathcal{R}$ and for $f=\sum_{R^{\prime} \in \mathcal{R}} h_{R^{\prime}} f_{R^{\prime}} \in L^{2}\left(\mathbb{T}^{2}\right)$, we have

$$
P_{R} f=P_{I} \otimes P_{J} f=\sum_{R^{\prime} \in \mathcal{R}, R^{\prime} \subseteq R} h_{R^{\prime}} f_{R^{\prime}}
$$

It is easy to see that for $R \in \mathcal{R}$ and $f \in L^{2}\left(\mathbb{T}^{2}\right)$,

$$
P_{R} f=\left(f-m_{I} f-m_{J} f+m_{I \times J}\right) \chi_{I \times J} .
$$

Recall that $g \in L^{2}(\mathbb{T})$ is said to belong to dyadic BMO, to be denoted $\mathrm{BMO}^{d}(\mathbb{T})$, if

$$
\sup _{I \in \mathcal{D}}\left(\frac{1}{|I|} \int_{I}\left|g(t)-m_{I} g\right|^{2} d t\right)^{1 / 2}<\infty .
$$

By John-Nirenberg's lemma, this is equivalent to

$$
\sup _{I \in \mathcal{D}}\left(\frac{1}{|I|} \int_{I}\left|g(t)-m_{I} g\right|^{p} d t\right)^{1 / p}<\infty
$$

for any $1 \leq p<\infty$.

Hence $g \in \mathrm{BMO}^{d}(\mathbb{T})$ if and only if there exists a constant $C$ such that for all $I \in \mathcal{D}$

$$
\sum_{I^{\prime} \in \mathcal{D}, I^{\prime} \subseteq I}\left|g_{I^{\prime}}\right|^{2} \leq C|I|^{1 / 2},
$$

or equivalently

$$
\sup _{I \in \mathcal{D}} \frac{1}{|I|^{1 / p}}\left\|P_{I} g\right\|_{p}<\infty
$$

for $1 \leq p<\infty$. 
The space BMO appears in many different contexts. We shall use that $\mathrm{BMO}^{\mathrm{d}}(\mathbb{T})=\left(H^{1, d}(\mathbb{T})\right)^{*}$ where $H^{1, d}$ is defined in terms of the dyadic square functions $\mathcal{S}$,

$$
\mathcal{S} g=\left(\sum_{I \in \mathcal{D}} \frac{\chi_{I}}{|I|}\left|g_{I}\right|^{2}\right)^{1 / 2}
$$

That is,

$$
H^{1, d}(\mathbb{T})=\left\{g \in L^{1}(\mathbb{T}): \mathcal{S} g \in L^{1}(\mathbb{T})\right\}
$$

Using Carleson measures, this gives rise to a description of $\mathrm{BMO}^{\mathrm{d}}$ in terms of symbols $g$ for which the dyadic paraproduct $\pi_{g}$,

$$
\pi_{g}(f)=\sum_{I \in \mathcal{D}} g_{I} m_{I} f h_{I}
$$

or its adjoint operator $\Delta_{g}, \Delta_{g}(f)=\sum_{I \in \mathcal{D}} g_{I} f_{I} \frac{\chi_{I}}{|I|}$, is bounded on $L^{2}(\mathbb{T})$ (or equivalently, on $L^{p}(\mathbb{T})$ for $\left.1<p<\infty\right)$.

The situation in two variables it is rather different and much more delicate. One main reason for the difficulties encountered in the multivariable theory is the failure of the naive generalization of the Carleson Embedding Theorem to several variables (see $[\mathrm{C}]$, [Fef]). The reader is referred to [ChFef2] for an overview on the theory and an outline of the main differences.

Several new results (e. g. [FS] and [PS]) further exhibit the differences between certain BMO spaces on the polydisk defined by multi-variable versions of the different yet equivalent characterizations of $\operatorname{BMO}(\mathbb{T})$.

A function $f \in L^{2}\left(\mathbb{T}^{2}\right)$ is said to belong to the rectangular dyadic BMO space, to be denoted $\mathrm{BMO}_{\text {rect }}^{d}$, if

$$
\sup _{R=I \times J \in \mathcal{R}}\left(\frac{1}{|R|} \int_{R}\left|f(t, s)-m_{I} f(s)-m_{J} f(t)+m_{I \times J} f\right|^{2} d t d s\right)^{1 / 2}<\infty .
$$

Or equivalently,

$$
\|\varphi\|_{\mathrm{BMO}_{\mathrm{rect}}^{d}}=\sup _{R \in \mathcal{R}} \frac{1}{|R|^{1 / 2}}\left\|P_{R} \varphi\right\|_{2} .
$$

We will also consider a $p$-version of the dyadic rectangular norm for $1 \leq p<\infty$

$$
\|\varphi\|_{\mathrm{BMO}_{\mathrm{rect}, p}^{d}}=\sup _{R \in \mathcal{R}} \frac{1}{|R|^{1 / p}}\left\|P_{R} \varphi\right\|_{p} .
$$

Here, $\|\cdot\|_{\mathrm{BMO}_{\text {rect }}^{d}}=\|\cdot\|_{\mathrm{BMO}_{\mathrm{rect}, 2}^{\mathrm{d}}}$. In the one-variable case, the corresponding norms are of course all equivalent because of John-Nirenberg's lemma. 
Let us start by defining $\mathrm{BMO}_{\text {prod }}^{d}\left(\mathbb{T}^{2}\right)$ as the dual of $H^{1, d}\left(\mathbb{T}^{2}\right)$, the space of functions $f \in L^{1}\left(\mathbb{T}^{2}\right)$ such that $\mathcal{S}(f) \in L^{1}\left(\mathbb{T}^{2}\right)$, where

$$
\mathcal{S}(f)=\left(\sum_{R \in \mathcal{R}}\left|f_{R}\right|^{2} h_{R}^{2}\right)^{1 / 2} .
$$

Although $\mathrm{BMO}_{\text {prod }}^{d}\left(\mathbb{T}^{2}\right)$ cannot be characterized by (1.3) [Fef], it was shown by Bernard in the dyadic case [Be] and also by Chang and R. Fefferman in a continuous version $[\mathrm{ChFef1}]$ that $\mathrm{BMO}_{\text {prod }}^{d}\left(\mathbb{T}^{2}\right)$ can also be described as the space of functions $\varphi \in L^{2}\left(\mathbb{T}^{2}\right)$ for which there exists $C>0$ such that

$$
\|\varphi\|_{\text {prod }}=\sup _{\Omega \subset \mathbb{T}^{2}} \frac{1}{|\Omega|^{1 / 2}}\left\|P_{\Omega} \varphi\right\|_{2}<\infty
$$

where the supremum is taken over all measurable sets $\Omega \subseteq \mathbb{T}^{2}$. This immediately implies $\mathrm{BMO}_{\text {prod }}^{d} \subseteq \mathrm{BMO}_{\text {rect }, 2}$.

The connection between both spaces can be also seen from the description of $\mathrm{BMO}_{\text {prod }}^{d}$ in terms of the boundedness of the dyadic paraproduct in two variables, defined by $\pi_{b}^{(1,2)}(f)=\sum_{R \in \mathcal{R}} b_{R} m_{R} f h_{R}$.

It follows from Chang's generalization of the Carleson Embedding Theorem (see [Ch]) that $b \in \mathrm{BMO}_{\text {prod }}^{d}$ if and only if the double paraproduct $\pi_{b}^{(1,2)}$ is bounded on $L^{2}\left(\mathbb{T}^{2}\right)$. In our paper the following fact will be rather crucial:

$$
\|\varphi\|_{\text {prod }} \approx\left\|\pi_{\varphi}^{(1,2)}\right\|
$$

An similar characterization for $\mathrm{BMO}_{\text {rect }}^{d}$ was proved in [PS], Proposition 3.3.1, namely that $b \in \mathrm{BMO}_{\text {rect }}^{d}$ if and only if $\pi_{b}^{(1,2)}$ maps $L^{2}(\mathbb{T}) \hat{\otimes} L^{2}(\mathbb{T})$ boundedly into $L^{2}\left(\mathbb{T}^{2}\right)$, where $L^{2}(\mathbb{T}) \hat{\otimes} L^{2}(\mathbb{T})$ stands for the projective tensor product. This also implies that $\mathrm{BMO}_{\text {prod }}^{d} \subsetneq \mathrm{BMO}_{\text {rect }}^{d}$ (see [Fef] for an alternative approach).

We shall try to better understand the difference between both spaces. Two approaches are used to this end. First we observe that John-Nirenberg type inequalities do not hold in $\mathrm{BMO}_{\text {rect }}^{d}$, in the sense that the 2-norm in the definition of $\mathrm{BMO}_{\text {rect }}^{d}$ cannot be replaced by any other $p$-norm. This solves a question left open in [FS]. Secondly, we analyse the behaviour of the sweep of functions in the $\mathrm{BMO}_{\text {prod }}^{d}$ and in the $\mathrm{BMO}_{\text {rect, } p}^{d}$ spaces.

Our main new tool will be characterizations of $\mathrm{BMO}_{\text {prod }}^{d}$ in terms of Haar multipliers. Recall that sequence of functions $\left(\phi_{R}\right)_{R \in \mathcal{R}}$ is called a Haar multiplier (see $(2.16)$ or $[\mathrm{Per}]$ ) on $L^{p}\left(\mathbb{T}^{2}\right)$, if the map

$$
f=\sum_{R \in \mathcal{R}} f_{R} h_{R}(t, s) \mapsto \sum_{R \in \mathcal{R}} \phi_{R}(t, s) f_{R} h_{R}(t, s)
$$

defines a bounded operator on $L^{p}\left(\mathbb{T}^{2}\right)$. 
We shall say that $b \in \mathrm{BMO}_{\text {mult }}^{d}$ if $\left\{\left(P_{R} b\right)\right\}_{R \in \mathcal{R}}$ defines a Haar multiplier on $L^{2}\left(\mathbb{T}^{2}\right)$.

Using the characterization of $\mathrm{BMO}_{\text {prod }}^{d}$ in terms of dyadic paraproducts, we observe that $b \in \mathrm{BMO}_{\text {prod }}^{d}$ if the operator $\Delta_{b}=\left(\pi_{\bar{b}}^{(1,2)}\right)^{*}$ given by $\Delta_{b}(f)=$ $\sum_{R} b_{R} h_{R} f_{R} h_{R}$ defines a bounded operator on $L^{2}\left(\mathbb{T}^{2}\right)$. Hence $b \in \mathrm{BMO}_{\text {prod }}^{d}$ if and only if $\left(b_{R} h_{R}\right)_{R \in \mathcal{R}}$ is a Haar multiplier on $L^{2}\left(\mathbb{T}^{2}\right)$.

On the other hand, letting the Haar multiplier $\left(P_{R^{\prime}} b\right)_{R^{\prime} \in \mathcal{R}}$ act on $h_{R}$, we see that $\frac{1}{|R|^{1 / 2}}\left\|P_{R} b\right\|_{2} \leq\|b\|_{\text {mult }}$, implying that $\mathrm{BMO}_{\text {mult }}^{d} \subseteq \mathrm{BMO}_{\text {rect }}^{d}$.

We shall get a description of $\mathrm{BMO}_{\text {mult }}^{d}$ in terms of the boundedness of the operator

$$
\Lambda_{b}=\pi_{b}^{(1,2)}+\left(\pi_{b}^{(1,2)}\right)^{*}+\Delta_{\pi_{b}}+\left(\Delta_{\pi_{b}}\right)^{*},
$$

where $\Delta_{\pi_{b}}$ (see Definition 2.4) is an operator combining the one-variable paraproduct $\pi$ and its adjoint. This will allow us to prove that $\mathrm{BMO}_{\text {mult }}^{d}\left(\mathbb{T}^{2}\right)$ $=\mathrm{BMO}_{\text {prod }}^{d}\left(\mathbb{T}^{2}\right)$ (see Theorem 2.8).

On the other hand, $\mathrm{BMO}_{\text {rect }}^{d}$ can also be described using $\Lambda_{b}$. We show that $\mathrm{BMO}_{\text {rect }}^{d}$ can be characterized in terms of "average boundedness" of $\Lambda_{b}$ or in terms of its boundedness from $L^{2}(\mathbb{T}) \hat{\otimes} L^{2}(\mathbb{T})$ into $L^{2}\left(\mathbb{T}^{2}\right)$.

The paper is divided into three sections. The first one is devoted to the introduction of the space $\mathrm{BMO}_{\text {mult }}^{d}$ and the proof of some of its properties. We see that $\mathrm{BMO}_{\text {mult }}^{d}$ can be characterized as the space of symbols $b$ for which the operator $\Lambda_{b}$ is bounded, and that this space coincides with $\mathrm{BMO}_{\text {prod }}^{d}$.

Section 3 deals with results on sweep functions. We prove the following formula connecting the boundedness of $\pi_{b}^{(1,2)}$ and $\Lambda_{S_{b}}$ (see Lemma 3.2):

$$
\pi_{b}^{(1,2)^{*}} \pi_{b}^{(1,2)}=\Lambda_{S_{b}}+D_{b}
$$

where $D_{b}$ is bounded if $b \in \mathrm{BMO}_{\text {rect, } 2}^{d}$.

This allows us to see that $b \in \mathrm{BMO}_{\text {prod }}^{d}$ if and only if $S_{b} \in \mathrm{BMO}_{\text {prod }}^{d}$. We also obtain a characterization of $\mathrm{BMO}_{\text {prod }}^{d}$ in terms of nested commutators with dyadic martingale transforms, sharpening a result from [PS].

We further use the formula (1.7) to quantify the difference between the BMO spaces we have considered, and to get a characterization of $\mathrm{BMO}_{\text {prod }}^{d}$ relying only upon the $\mathrm{BMO}_{\text {rect }}^{d}$ norm of the $n$-fold sweeps.

Finally, in the last section, we apply the results from the previous ones together with interpolation to study the scale of spaces $\mathrm{BMO}_{\text {rect, } p}^{d}$ introduced in (1.4) and show that these spaces are pairwise distinct. As a corollary, we obtain that $\mathrm{BMO}_{\text {prod }}^{d} \subsetneq \cap_{p \geq 1} \mathrm{BMO}_{\text {rect }, p}^{d}$. 


\section{BMO via Haar multipliers}

Definition 2.1 We shall say that $b \in \mathrm{BMO}_{\text {mult }}^{d}$ if $\left\{\left(P_{R} b\right)\right\}_{R \in \mathcal{R}}$ defines a Haar multiplier on $L^{2}\left(\mathbb{T}^{2}\right)$, i.e. there exists $C>0$ such that

$$
\left\|\sum_{R \in \mathcal{R}} P_{R} b f_{R} h_{R}\right\|_{2} \leq C\|f\|_{2}
$$

for all $f \in L^{2}\left(\mathbb{T}^{2}\right)$. We define $\|b\|_{\text {mult }}$ as the norm of the corresponding operator.

Let us start by pointing out some simple facts about this space.

Given $I \in \mathcal{D}$ we write $P_{I}$ for the operator on $L^{2}(\mathbb{T})$ given by $(1.1)$, and $\tilde{P}_{I}=P_{I} \otimes$ id for the corresponding projection on $L^{2}\left(\mathbb{T}^{2}\right)$,

$$
\tilde{P}_{I}(f)(t, s)=\sum_{I^{\prime} \in \mathcal{D}, I^{\prime} \subseteq I} h_{I^{\prime}}(t) f_{I^{\prime}}(s) .
$$

Similarly, given $J \in \mathcal{D}_{2}$, we write $\tilde{P}_{J}$ for id $\otimes P_{J}$.

Of course, $\tilde{P}_{I}(f)(t, s)=P_{I}(f(s, \cdot))(t)$ and $P_{R} f=\tilde{P}_{J}\left(\tilde{P}_{I} f\right)$ for $R=I \times J$.

\section{Proposition 2.2}

$$
\begin{gathered}
L^{\infty}\left(\mathbb{T}^{2}\right) \subset \mathrm{BMO}_{\text {mult }}^{d}\left(\mathbb{T}^{2}\right) \\
\operatorname{BMO}^{\mathrm{d}}(\mathbb{T}) \otimes \mathrm{BMO}^{\mathrm{d}}(\mathbb{T}) \subseteq \mathrm{BMO}_{\text {mult }}^{d}\left(\mathbb{T}^{2}\right)
\end{gathered}
$$

Proof. Using (1.2), one easily obtains the following formula:

$$
\sum_{R \in \mathcal{R}} P_{R} b f_{R} h_{R}=f b-\sum_{I \in \mathcal{D}}\left(m_{I} b\right) f_{I} h_{I}-\sum_{J \in \mathcal{D}}\left(m_{J} b\right) f_{J} h_{J}+\sum_{R \in \mathcal{R}} m_{R} b f_{R} h_{R} .
$$

Now (2.1) follows from this expression together with

$$
\left\|\sum_{J \in \mathcal{D}}\left(m_{J} b\right) f_{J} h_{J}\right\|_{L^{2}\left(\mathbb{T}^{2}\right)}^{2}=\sum_{J \in \mathcal{D}}\left\|m_{J} b f_{J}\right\|_{L^{2}(\mathbb{T})}^{2},
$$

since

$$
\left\|\sum_{J \in \mathcal{D}}\left(m_{J} b\right) f_{J} h_{J}\right\|_{L^{2}\left(\mathbb{T}^{2}\right)}^{2} \leq\|b\|_{\infty}^{2} \sum_{J \in \mathcal{D}}\left\|f_{J}\right\|_{L^{2}(\mathbb{T})}^{2}=\|b\|_{\infty}^{2}\|f\|_{2}^{2},
$$

and the trivial estimates for the terms $b f$ and $\sum_{R \in \mathcal{R}} m_{R} b f_{R} h_{R}$.

To see (2.2), note first that for $b_{1} \in \mathrm{BMO}^{\mathrm{d}}$ and $f \in L^{2}(\mathbb{T})$,

$$
\sum_{I \in \mathcal{D}} P_{I} b_{1} f_{I} h_{I}=\left(\pi_{b_{1}}+\Delta_{b_{1}}\right) f=\left(\pi_{b_{1}}+\left(\pi_{\bar{b}_{1}}\right)^{*}\right) f .
$$

Therefore $\left(P_{I} b_{1}\right)_{I \in \mathcal{D}}$ defines a bounded Haar multiplier on $L^{2}(\mathbb{T})$. 
Now let $b(t, s)=b_{1}(t) b_{2}(s)$ with $b_{1}, b_{2} \in \operatorname{BMO}^{\mathrm{d}}(\mathbb{T})$. Then $P_{R}(b)=$ $P_{I}\left(b_{1}\right) P_{J}\left(b_{2}\right)$ and therefore

$$
\sum_{R \in \mathcal{R}} P_{R} b f_{R} h_{R}=\sum_{I \in \mathcal{D}} P_{I} b_{1}\left(\sum_{J \in \mathcal{D}} P_{J} b_{2} f_{J} h_{J}\right)_{I} h_{I}
$$

This yields

$$
\begin{aligned}
\left\|\sum_{R \in \mathcal{R}} P_{R} b f_{R} h_{R}\right\|_{2}^{2} & =\int_{\mathbb{T}^{2}}\left|\sum_{I \in \mathcal{D}} P_{I} b_{1}(t)\left(\sum_{J \in \mathcal{D}} P_{J} b_{2} f_{J} h_{J}\right)_{I}(s) h_{I}(t)\right|_{2}^{2} d t d s \\
& \leq C\left\|b_{1}\right\|_{\mathrm{BMO}}^{2} \int_{\mathbb{T}} \sum_{I \in \mathcal{D}}\left|\sum_{J \in \mathcal{D}}\left(P_{J} b_{2} f_{J} h_{J}\right)_{I}(s)\right|^{2} d s \\
& \leq C^{2}\left\|b_{1}\right\|_{\mathrm{BMO}}^{2}\left\|b_{2}\right\|_{\mathrm{BMO}}^{2} \sum_{I \in \mathcal{D}} \sum_{J \in \mathcal{D}}\left|f_{I \times J}\right|^{2}
\end{aligned}
$$

with some absolute constant $C>0$.

As announced in the introduction, we first relate this space to $\mathrm{BMO}_{\text {prod }}^{d}$. For this purpose we introduce the dyadic paraproducts in two variables (see $[\mathrm{PS}])$ :

Definition 2.3 Given $b \in L^{2}\left(\mathbb{T}^{2}\right)$, we write

$$
\pi_{b}^{(1,2)}(f)=\sum_{R \in \mathcal{R}} b_{R} m_{R} f h_{R}
$$

and

$$
\Delta_{b}^{(1,2)}(f)=\left(\pi_{\bar{b}}^{(1,2)}\right)^{*}(f)=\sum_{R \in \mathcal{R}} b_{R} f_{R} \frac{\chi_{R}}{|R|}
$$

The formula

$$
\left\langle\pi_{b}^{(1,2)}(f), g\right\rangle=\left\langle f, \Delta_{\bar{b}}^{(1,2)}(g)\right\rangle=\int_{\mathbb{T}^{2}} b\left(\sum_{R \in \mathcal{R}} m_{R}(f) \bar{g}_{R} h_{R}\right) d t d s
$$

for $f, g \in \mathcal{H}_{00}$ completely describe the action of the operators $\pi_{b}^{(1,2)}$ and $\Delta_{b}^{(1,2)}$.

Let us now define the following mixed operators (see $[\mathrm{PS}]$ ).

Definition 2.4 Given $b \in L^{2}\left(\mathbb{T}^{2}\right)$, we define the operators $\pi_{\Delta_{b}}$ and $\Delta_{\pi_{b}}$ by

$$
\left\langle\pi_{\Delta_{b}}(f), g\right\rangle=\left\langle f, \Delta_{\pi_{\bar{b}}}(g)\right\rangle=\int_{\mathbb{T}^{2}} b\left(\sum_{I \times J \in \mathcal{R}} m_{I}\left(f_{J}\right) m_{J}\left(\bar{g}_{I}\right) h_{I \times J}\right) d t d s
$$

for $f, g \in \mathcal{H}_{00}$.

We write

$$
\Lambda_{b}=\pi_{b}^{(1,2)}+\Delta_{b}^{(1,2)}+\Delta_{\pi_{b}}+\pi_{\Delta_{b}} .
$$


Clearly we have the following expressions:

$$
\begin{aligned}
\pi_{b}^{(1,2)}(f)(t, s) & =\sum_{I \in \mathcal{D}} \pi_{b_{I}}\left(m_{I} f\right)(s) h_{I}(t) \\
\Delta_{b}^{(1,2)}(f)(t, s) & =\sum_{I \in \mathcal{D}} \Delta_{b_{I}}\left(f_{I}\right)(s) h_{I}^{2}(t) \\
\Delta_{\pi_{b}}(f)(t, s) & =\sum_{I \in \mathcal{D}} \pi_{b_{I}}\left(f_{I}\right)(s) h_{I}^{2}(t) \\
\pi_{\Delta_{b}}(f)(t, s) & =\sum_{I \in \mathcal{D}} \Delta_{b_{I}}\left(m_{I} f\right)(s) h_{I}(t) .
\end{aligned}
$$

Lemma 2.5 Let $R=I \times J \in \mathcal{R}$ and denote $R_{+}=I^{+} \times J^{+} \cup I^{-} \times J^{-}$and $R_{-}=I^{+} \times J^{-} \cup I^{-} \times J^{+}$. Then

$$
\pi_{b}^{(1,2)}\left(h_{R}\right)=\left(P_{R_{+}}(b)+P_{R_{-}}(b)\right) h_{R}=\left(P_{R_{+}}(b)-P_{R_{-}}(b)\right)|R|^{-1 / 2}
$$

Proof. Using that $m_{R^{\prime}}\left(h_{R}\right) \neq 0$ only if $I^{\prime} \subsetneq I$ and $J^{\prime} \subsetneq J$ and that in this case $m_{R^{\prime}}\left(h_{R}\right)=h_{R}\left(x_{R^{\prime}}\right)$, where $x_{R^{\prime}}=\left(t_{I^{\prime}}, s_{J^{\prime}}\right)$ is the center of $R$, we obtain that

$$
\pi_{b}^{(1,2)}\left(h_{R}\right)=\sum_{I^{\prime} \subsetneq I, J^{\prime} \subsetneq J} b_{R^{\prime}} h_{R}\left(x_{R^{\prime}}\right) h_{R}^{\prime}
$$

Observe that $h_{R}\left(x_{R^{\prime}}\right)=h_{R}(t, s)=\frac{1}{|R|^{1 / 2}}$ for $R^{\prime} \subset R_{+}$and $(t, s) \in R^{+}$. Similarly $h_{R}\left(x_{R^{\prime}}\right)=h_{R}(t, s)=-\frac{1}{|R|^{1 / 2}}$ for $R^{\prime} \subset R_{+}$and $(t, s) \in R^{-}$. This gives (2.10).

Corollary 2.6 Let $b \in L^{2}\left(\mathbb{T}^{2}\right)$. Then $b \in \mathrm{BMO}_{\text {prod }}^{d}$ if and only if $\left(P_{R_{+}}(b)+\right.$ $\left.\left.P_{R_{-}}(b)\right) h_{R}\right)_{R \in \mathcal{R}}$ is a Haar multiplier on $L^{2}\left(\mathbb{T}^{2}\right)$.

Lemma 2.7 $\Lambda_{b}(f)=\sum_{R \in \mathcal{R}} P_{R}(b) f_{R} h_{R}$.

Proof. Note that for $\phi, g \in \mathcal{H}_{00}$, we have

$$
\phi g=\pi_{\phi}(g)+\Delta_{\phi}(g)+\pi_{g}(\phi) .
$$

As in (2.3), one obtains

$$
\sum_{J \in \mathcal{D}} P_{J}(\phi) g_{J} h_{J}=\pi_{\phi}(g)+\Delta_{\phi}(g) .
$$


Combining (2.12) with the formulas in (2.6)- (2.9) we get

$$
\begin{aligned}
& \Lambda_{b}(f)(t, s)=\sum_{I \in \mathcal{D}}\left(\pi_{b_{I}}+\Delta_{b_{I}}\right)\left(m_{I} f\right)(s) h_{I}(t)+\sum_{I \in \mathcal{D}}\left(\pi_{b_{I}}+\Delta_{b_{I}}\right)\left(f_{I}\right)(s) h_{I}^{2}(t) \\
& =\sum_{I \in \mathcal{D}} \sum_{J \in \mathcal{D}} P_{J}\left(b_{I}\right)(s)\left(m_{I} f\right)_{J} h_{J}(s) h_{I}(t)+\sum_{I \in \mathcal{D}} \sum_{J \in \mathcal{D}} P_{J}\left(b_{I}\right)(s)\left(f_{I}\right)_{J} h_{J}(s) h_{I}^{2}(t) \\
& =\sum_{J \in \mathcal{D}}\left(\sum_{I \in \mathcal{D}}\left(\tilde{P}_{J} b\right)_{I}(s) m_{I}\left(f_{J}\right) h_{I}(t)\right) h_{J}(s)+\sum_{J \in \mathcal{D}}\left(\sum_{I \in \mathcal{D}}\left(\tilde{P}_{J} b\right)_{I}(s)\left(f_{J}\right)_{I} h_{I}^{2}(t)\right) h_{J}(s) \\
& =\sum_{J \in \mathcal{D}}\left(\pi_{\tilde{P}_{J} b(s, \cdot)}\left(f_{J}\right)\right)(t) h_{J}(s)+\sum_{J \in \mathcal{D}}\left(\Delta_{\tilde{P}_{J} b(s, \cdot)}\left(f_{J}\right)\right)(t) h_{J}(s) \\
& =\sum_{I \times J \in \mathcal{R}} \tilde{P}_{I}\left(\tilde{P}_{J}(b)\right)(t, s) f_{I \times J} h_{I \times J}(t, s) \\
& =\sum_{R \in \mathcal{R}} P_{R}(b)(t, s) f_{R} h_{R}(t, s) .
\end{aligned}
$$

We now are ready to prove our characterization of $\mathrm{BMO}_{\text {prod }}^{d}$ in terms of Haar multipliers.

Theorem 2.8 $\mathrm{BMO}_{\text {prod }}^{d}=\mathrm{BMO}_{\text {mult }}^{d}$.

Proof. To see that $\mathrm{BMO}_{\text {prod }}^{d} \subseteq \mathrm{BMO}_{\text {mult }}^{d}$, it suffices to see that the boundedness of $\pi_{b}^{(1,2)}$ implies the boundedness of $\Delta_{\pi_{b}}$. This was proved in [PS], we include here a proof for the sake of completeness.

By (2.5) and the characterization of $\mathrm{BMO}_{\text {prod }}^{d}$ as the dual of $H^{1, d}$, the space of functions with integrable square function, we simply need to show that

$$
F=\sum_{I \times J \in \mathcal{R}} m_{I}\left(f_{J}\right) m_{J}\left(g_{I}\right) h_{I \times J}(t, s)
$$

belongs to $H^{1, d}$. Note that

$$
\mathcal{S}(F)(t, s)=\left(\sum_{I \times J \in \mathcal{R}}\left|m_{I}\left(f_{J}\right)\right|^{2}\left|m_{J}\left(g_{I}\right)\right|^{2} h_{I \times J}^{2}(t, s)\right)^{1 / 2} .
$$

Therefore

$$
\begin{aligned}
\mathcal{S}(F)(t, s) & \leq\left(\sum_{J \in \mathcal{D}} \sum_{I \in \mathcal{D}}\left(g_{I}^{*}(s)\right)^{2} h_{J}^{2}(s)\left(f_{J}^{*}(t)\right)^{2} h_{I}^{2}(t)\right)^{1 / 2} \\
& =\left(\sum_{I \in \mathcal{D}}\left(g_{I}^{*}(s)\right)^{2} h_{I}^{2}(t)\right)^{1 / 2}\left(\sum_{J \in \mathcal{D}}\left(f_{J}^{*}(t)\right)^{2} h_{J}^{2}(s)\right)^{1 / 2},
\end{aligned}
$$


and hence

$$
\begin{array}{rl}
\int_{\mathbb{T}^{2}} & \mathcal{S}(F)(t, s) d t d s \leq \\
\leq & \left(\int_{\mathbb{T}^{2}} \sum_{I \in \mathcal{D}}\left(g_{I}^{*}(s)\right)^{2} h_{I}^{2}(t) d t d s\right)^{1 / 2}\left(\int_{\mathbb{T}^{2}} \sum_{J \in \mathcal{D}}\left(f_{J}^{*}(t)\right)^{2} h_{J}^{2}(s) d t d s\right)^{1 / 2} \\
& =\left(\sum_{I \in \mathcal{D}} \int_{\mathbb{T}}\left(g_{I}^{*}(s)\right)^{2} d s\right)^{1 / 2}\left(\sum_{J \in \mathcal{D}} \int_{\mathbb{T}}\left(f_{J}^{*}(t)\right)^{2} d t\right)^{1 / 2} \\
& \leq C\left(\sum_{I \in \mathcal{D}}\left\|g_{I}\right\|_{2}^{2}\right)^{1 / 2}\left(\sum_{J \in \mathcal{D}}\left\|f_{J}\right\|_{2}^{2}\right)^{1 / 2}=C\|g\|_{2}\|f\|_{2} .
\end{array}
$$

To prove the reverse inclusion $\mathrm{BMO}_{\text {mult }}^{d} \subseteq \mathrm{BMO}_{\text {prod }}^{d}$, we shall use the characterization of $\mathrm{BMO}_{\text {prod }}^{d}$ given in (1.6).

It is clear that for each measurable set $\Omega$, we have $P_{\Omega}(b)=P_{\Omega}\left(\pi_{b}^{(1,2)}\left(\chi_{\Omega}\right)\right)$. We shall show now that

$$
P_{\Omega}\left(\pi_{b}^{(1,2)}\left(\chi_{\Omega}\right)\right)=P_{\Omega}\left(\Lambda_{b}\left(\chi_{\Omega}\right)\right) .
$$

Let $R \in \mathcal{R}$ and $R \subseteq \Omega$. Then by (2.10)

$$
\left\langle\Delta_{b}^{(1,2)}\left(\chi_{\Omega}\right), h_{R}\right\rangle=\left\langle\chi_{\Omega}, \pi_{b}^{(1,2)}\left(h_{R}\right)\right\rangle=|R|^{-1 / 2}\left\langle\chi_{\Omega}, P_{R^{+}} b-P_{R^{-}} b\right\rangle=0 .
$$

This shows that $P_{\Omega}\left(\Delta_{b}^{(1,2)}\left(\chi_{\Omega}\right)\right)=0$.

On the other hand, we also have for $R=I \times J \subseteq \Omega$ that

$$
\pi_{\Delta_{b}}\left(h_{R}\right)=\sum_{I^{\prime} \subsetneq I} b_{I^{\prime} \times J} m_{I^{\prime}}\left(h_{I}\right) \chi_{J} h_{I^{\prime}}
$$

Using that $\left\langle\chi_{\Omega}, \chi_{J} h_{I^{\prime}}\right\rangle=0$ for all $I^{\prime} \subseteq I$, we obtain $P_{\Omega}\left(\pi_{\Delta_{b}}\left(\chi_{\Omega}\right)\right)=0$.

Similarly, $P_{\Omega}\left(\Delta_{\pi_{b}}\left(\chi_{\Omega}\right)\right)=0$. Finally,

$$
\begin{aligned}
\left\|P_{\Omega}(b)\right\| & =\left\|P_{\Omega}\left(\pi_{b}^{(1,2)}\left(\chi_{\Omega}\right)\right)\right\|=\left\|P_{\Omega}\left(\Lambda_{b}\left(\chi_{\Omega}\right)\right)\right\| \\
& \left.\leq \| \Lambda_{b}\left(\chi_{\Omega}\right)\right)\|\leq\| \Lambda_{b}|\| \Omega|^{1 / 2} .
\end{aligned}
$$

As a consequence of Thm 2.8, we can sharpen Thm 7.7.2 from [PS] and characterize $\mathrm{BMO}_{\text {prod }}^{d}$ in terms of the boundedness of nested commutators with dyadic martingale transforms. This can be understood as a dyadic analogue of the characterization of the continuous product $\mathrm{BMO}$ space $\mathrm{BMO}_{\text {prod }}$ as the space of functions for which the nested commutator

$$
\left[H_{1},\left[H_{2}, b\right]\right]: L^{2}\left(\mathbb{T}^{2}\right) \rightarrow L^{2}\left(\mathbb{T}^{2}\right)
$$

is bounded, where $H_{1}$ resp. $H_{2}$ denote the Hilbert transform in the first resp. second variable on $L^{2}\left(\mathbb{T}^{2}\right)$. The latter was proved in [FS] and [LF]. 
Let $\Sigma_{1}, \Sigma_{2}$ be the spaces of all sequences of signs indexed by the elements of $\mathcal{D}_{1}, \mathcal{D}_{2}, \Sigma_{1}=\{0,1\}^{\mathcal{D}_{1}}, \Sigma_{1}=\{0,1\}^{\mathcal{D}_{2}}$, and let $d \sigma_{1}$ denote the natural product probability measure on $\Sigma_{1}$, which assigns measure $2^{-n}$ to each cylindrical set of length $n$. Let $d \sigma_{2}$ denote the corresponding measure on $\Sigma_{2}$. Let $\Sigma=\Sigma_{1} \times \Sigma_{2}$, with $d \sigma$ denoting the product measure, and $\mathcal{R}=\mathcal{D}_{1} \times \mathcal{D}_{2}$ as before.

For $\sigma_{1}=\left(\sigma_{1}(I)\right)_{I \in \mathcal{D}_{1}} \in \Sigma_{1}, \sigma_{2}=\left(\sigma_{2}(J)\right)_{J \in \mathcal{D}_{2}} \in \Sigma_{2}$, let $T_{\sigma_{1}}, T_{\sigma_{2}}$ denote the dyadic martingale transforms

$$
\begin{aligned}
& T_{\sigma_{1}}: L^{2}\left(\mathbb{T}^{2}\right) \rightarrow L^{2}\left(\mathbb{T}^{2}\right), \quad f=\sum_{I \times J \in \mathcal{R}} f_{I \times J} h_{I \times J} \mapsto \sum_{I \times J \in \mathcal{R}} \sigma_{1}(I) f_{I \times J} h_{I \times J}, \\
& T_{\sigma_{2}}: L^{2}\left(\mathbb{T}^{2}\right) \rightarrow L^{2}\left(\mathbb{T}^{2}\right), \quad f=\sum_{I \times J \in \mathcal{R}} f_{I \times J} h_{I \times J} \mapsto \sum_{I \times J \in \mathcal{R}} \sigma_{2}(J) f_{I \times J} h_{I \times J} .
\end{aligned}
$$

Theorem 2.9 Let $b \in L^{2}\left(\mathbb{T}^{2}\right)$. Then the following are equivalent:

(i) $b \in \mathrm{BMO}_{\text {prod }}^{d}$

(ii) The nested commutators

$$
\left[T_{\sigma_{1}},\left[T_{\sigma_{2}}, b\right]\right]: L^{2}\left(\mathbb{T}^{2}\right) \rightarrow L^{2}\left(\mathbb{T}^{2}\right)
$$

are uniformly bounded for all $\sigma_{1} \in \Sigma_{1}, \sigma_{2} \in \Sigma_{2}$.

(iii) The nested commutators $\left[T_{\sigma_{1}},\left[T_{\sigma_{2}}, b\right]\right]: L^{2}\left(\mathbb{T}^{2}\right) \rightarrow L^{2}\left(\mathbb{T}^{2}\right)$ are bounded on average, in the sense that the map

$$
\Phi_{b}: L^{2}\left(\mathbb{T}^{2}\right) \rightarrow L^{2}\left(\Sigma_{1} \times \Sigma_{2} \times \mathbb{T}^{2}\right), f \mapsto\left[T_{\sigma_{1}},\left[T_{\sigma_{2}}, b\right]\right] f
$$

is bounded.

In this case, we have

$$
\|b\|_{\mathrm{BMO}_{\mathrm{prod}}^{d}} \approx\left\|\Lambda_{b}\right\| \leq\left\|\Phi_{b}\right\| \leq \sup _{\sigma_{1} \in \Sigma_{1}, \sigma_{2} \in \Sigma_{2}}\left\|\left[T_{\sigma_{1}},\left[T_{\sigma_{2}}, b\right]\right]\right\| \leq 4\left\|\Lambda_{b}\right\| .
$$

Proof. We use the ideas of the proofs of Thm 3.4, Cor 4.1 in [GPTV], adapted to the two-variable case, and of Thm 7.7.2 in [PS].

From [PS, p. 493], we know that

$$
\left[T_{\sigma_{1}},\left[T_{\sigma_{2}}, b\right]\right]=\left[T_{\sigma_{1}},\left[T_{\sigma_{2}}, \Lambda_{b}\right]\right] .
$$

Therefore

$$
\sup _{\sigma_{1} \in \Sigma_{1}, \sigma_{2} \in \Sigma_{2}}\left\|\left[T_{\sigma_{1}},\left[T_{\sigma_{2}}, b\right]\right]\right\| \leq 4\left\|\Lambda_{b}\right\| .
$$

The second inequality in (2.14) is obvious. 
Finally, for $f \in L^{2}\left(\mathbb{T}^{2}\right)$ one has

$$
\begin{aligned}
\left\|\Phi_{b} f\right\|^{2} & =\iint_{\Sigma_{1} \times \Sigma_{2}}\left\|\left[T_{\sigma_{1}},\left[T_{\sigma_{2}}, b\right]\right] f\right\|_{L^{2}\left(\mathbb{T}^{2}\right)}^{2} d \sigma_{1} d \sigma_{2} \\
& =\iint_{\Sigma_{1} \times \Sigma_{2}}\left\|\sum_{I \times J \in \mathcal{R}} \sigma_{1}(I) \sigma_{2}(J)\left[\tilde{P}_{I},\left[\tilde{P}_{J}, b\right]\right] f\right\|_{L^{2}\left(\mathbb{T}^{2}\right)}^{2} d \sigma_{1} d \sigma_{2} \\
& =\sum_{I \times J \in \mathcal{R}}\left\|\left[\tilde{P}_{I},\left[\tilde{P}_{J}, b\right]\right] f\right\|_{L^{2}\left(\mathbb{T}^{2}\right)}^{2} \\
& =\sum_{I \times J \in \mathcal{R}}\left\|\left[\tilde{P}_{I},\left[\tilde{P}_{J}, \Lambda_{b}\right]\right] f\right\|_{L^{2}\left(\mathbb{T}^{2}\right)}^{2}\left\|\left(\tilde{P}_{I} \tilde{P}_{J} \Lambda_{b}-\tilde{P}_{I} \Lambda_{b} \tilde{P}_{J}-\tilde{P}_{J} \Lambda_{b} \tilde{P}_{I}+\Lambda_{b} \tilde{P}_{J} \tilde{P}_{I}\right) f\right\|_{L^{2}\left(\mathbb{T}^{2}\right)}^{2} \\
& =\sum_{I \times J \in \mathcal{R}}\left\|\tilde{P}_{I} \tilde{P}_{J} \Lambda_{b} f\right\|^{2}=\left\|\Lambda_{b} f\right\|^{2}, \\
& \geq \sum_{I \times J \in \mathcal{R}}
\end{aligned}
$$

since $\tilde{P}_{I} \Lambda_{b} \tilde{P}_{I}=0$ and $\tilde{P}_{J} \Lambda_{b} \tilde{P}_{J}=0$. This proves the first inequality in (2.14).

The martingale transformation approach is also interesting in the study of $\mathrm{BMO}_{\text {rect }}^{d}$. Although $\Lambda_{b}$ is in general not bounded for $b \in \mathrm{BMO}_{\text {rect }}^{d}$, the space $\mathrm{BMO}_{\text {rect }}^{d}$ can be characterized in terms of "average boundedness" of $\Lambda_{b}$, and also in terms of the boundedness of $\Lambda_{b}$ from $L^{2}(\mathbb{T}) \hat{\otimes} L^{2}(\mathbb{T})$ into $L^{2}\left(\mathbb{T}^{2}\right)$. For $\sigma=\left(\sigma_{1}, \sigma_{2}\right) \in \Sigma$, let $T_{\sigma}=T_{\sigma_{1}} T_{\sigma_{2}}: L^{2}\left(\mathbb{T}^{2}\right) \rightarrow L^{2}\left(\mathbb{T}^{2}\right)$.

Theorem 2.10 For $\varphi \in \mathcal{H}_{00},\|\varphi\|_{\mathrm{BMO}_{\text {rect }}^{d}}$ is equal to the norm of the operator

$$
\Psi_{\varphi}: L^{2}\left(\mathbb{T}^{2}\right) \rightarrow L^{2}\left(\mathbb{T}^{2} \times \Sigma\right), f \mapsto \Lambda_{\varphi} T_{\sigma} f .
$$

Proof. Let $f \in L^{2}\left(\mathbb{T}^{2}\right)$ and $\varphi \in \mathcal{H}_{00}$. From Lemma 2.7 we have

$$
\Lambda_{\varphi} f=\sum_{R \in \mathcal{R}} P_{R} \varphi f_{R} h_{R}
$$

Thus

$$
\begin{aligned}
\int_{\Sigma} \int_{\mathbb{T}^{2}}\left\|\Lambda_{\varphi} T_{\sigma} f\right\|^{2} d t d s d \sigma & \left.=\int_{\mathbb{T}^{2}} \int_{\Sigma} \| \sum_{R \in \mathcal{R}} \sigma(R)\left(P_{R} \varphi\right)(t, s)\right) f_{R} h_{R}(t, s) \|^{2} d \sigma d t d s \\
& =\int_{\mathbb{T}^{2}} \sum_{R \in \mathcal{R}}\left|f_{R}\right|^{2} \frac{\chi_{R}(t, s)}{|R|}\left|\left(P_{R} \varphi\right)(t, s)\right|^{2} d t d s \\
& =\sum_{R \in \mathcal{R}}\left|f_{R}\right|^{2} \frac{1}{|R|}\left\|P_{R} \varphi\right\|^{2} .
\end{aligned}
$$

Thus the operator norm of $\Psi_{\varphi}$ is $\sup _{R \in \mathcal{R}} \frac{1}{|R|^{1 / 2}}\left\|P_{R} \varphi\right\|=\|\varphi\|_{\mathrm{BMO}_{\text {rect }}^{d}}$. 
Proposition 2.11 If $b \in \mathrm{BMO}_{\text {rect }}^{d}$ then $\Lambda_{b}$ maps $L^{2}(\mathbb{T}) \hat{\otimes} L^{2}(\mathbb{T})$ into $L^{2}\left(\mathbb{T}^{2}\right)$.

Proof. Assume $f(t, s)=f_{1}(t) f_{2}(s)$ with $\left\|f_{1}\right\|=\left\|f_{2}\right\|=1$. Then we have

$$
\sum_{R \in \mathcal{R}} P_{R} b f_{R} h_{R}=\sum_{I \in \mathcal{D}} \tilde{P}_{I}\left(\sum_{J \in \mathcal{D}} \tilde{P}_{J} b\left(f_{2}\right)_{J} h_{J}\right)(t)\left(f_{1}\right)_{I} h_{I}(t)
$$

Writing $g(t, s)=\sum_{J \in \mathcal{D}} P_{J}(b(t, \cdot))(s)\left(f_{2}\right)_{J} h_{J}(s)$, we obtain

$$
\left\|\sum_{R \in \mathcal{R}} P_{R} b f_{R} h_{R}\right\|_{2}^{2}=\int_{\mathbb{T}} \int_{\mathbb{T}}\left|\sum_{I \in \mathcal{D}} P_{I}(g(\cdot, s))(t)\left(f_{1}\right)_{I} h_{I}(t)\right|^{2} d t d s .
$$

Now let us consider $g$ as a function in $t$ taking values in the Hilbert space $L^{2}(\mathbb{T})$. Recall that as in the scalar case, the Haar multiplier norm of $\left(P_{I} g\right)_{I \in \mathcal{D}}$ is controlled by the vector $\mathrm{BMO}^{\mathrm{d}}(\mathbb{T})$ norm of $g$ given by

$$
\sup _{I \in \mathcal{D}} \frac{1}{|I|^{1 / 2}}\left\|P_{I} g\right\|_{L^{2}\left(\mathbb{T}, L^{2}(\mathbb{T})\right)}
$$

Thus

$$
\sup _{\left\|f_{1}\right\|=1} \int_{\mathbb{T}}\left\|\sum_{I \in \mathcal{D}} P_{I}(g(\cdot, s))(t)\left(f_{1}\right)_{I} h_{I}(t)\right\|_{L^{2}(\mathbb{T})}^{2} d t \leq C \sup _{I} \frac{1}{|I|}\left\|P_{I} g\right\|_{L^{2}\left(\mathbb{T}, L^{2}(\mathbb{T})\right)}^{2} .
$$

Notice now that

$$
P_{I}\left(\sum_{J \in \mathcal{D}} P_{J}(b(t, \cdot))(s)\left(f_{2}\right)_{J} h_{J}(s)\right)=\sum_{J \in \mathcal{D}} P_{I \times J}(b)(t, s)\left(f_{2}\right)_{J} h_{J}(s) .
$$

On the other hand, applying a corresponding argument to the function $\left(\tilde{P}_{I} b\right)(t, s)=\sum_{J \in \mathcal{D}}\left(P_{I \times J} b\right)(t, s) h_{J}(s)$ understood as a function in $s$ which takes values in $L^{2}(\mathbb{T})$, we obtain for $\left\|f_{2}\right\|_{2}=1$

$$
\begin{aligned}
\left\|P_{I} g\right\|_{L^{2}\left(\mathbb{T}, L^{2}(\mathbb{T})\right)}^{2} & =\int_{\mathbb{T}^{2}}\left|\sum_{J \in \mathcal{D}} P_{I \times J}(b)(t, s)\left(f_{2}\right)_{J} h_{J}(s)\right|^{2} d t d s \\
& =\int_{\mathbb{T}}\left\|\sum_{J \in \mathcal{D}} P_{I \times J}(b)(\cdot, s)\left(f_{2}\right)_{J} h_{J}(s)\right\|_{L^{2}(\mathbb{T})}^{2} d s \\
& \leq C \sup _{J} \frac{1}{|J|}\left\|P_{I \times J}(b)\right\|_{L^{2}\left(\mathbb{T}, L^{2}(\mathbb{T})\right)}^{2} \leq C\|I \mid\| b \|_{\mathrm{BMO}_{\text {rect }}^{d}}^{2} .
\end{aligned}
$$

This finishes the proof of the proposition. 


\section{Sweeps of functions in BMO}

Let us now recall that the (dyadic) sweep of a function $\varphi \in L^{2}\left(\mathbb{T}^{2}\right)$ is defined as follows:

$$
S_{\varphi}=\sum_{R \in \mathcal{R}}\left|\varphi_{R}\right|^{2} \frac{\chi_{R}}{|R|}
$$

i.e. $S_{\varphi}=\mathcal{S}(\varphi)^{2}$.

We list some properties of the sweep which will be relevant for our purposes, the proofs of which are elementary and left to the reader.

\section{Proposition 3.1}

(i) $S_{\varphi}(t, s)=\sum_{I \in \mathcal{D}} S_{\varphi_{I}}(s) \frac{\chi_{I}(t)}{|I|}$.

(ii) $S_{\varphi}=\Delta_{\varphi}^{(1,2)}(\varphi)$.

(iii) $P_{\Omega}\left(S_{\varphi}\right)=P_{\Omega}\left(S_{P_{\Omega} \varphi}\right)$.

(iv) If $p>\frac{1}{2}$ then $\varphi \in L^{2 p}\left(\mathbb{T}^{2}\right)$ if and only if $S_{\varphi} \in L^{p}\left(\mathbb{T}^{2}\right)$.

(v) If $S_{\varphi} \in L^{\infty}$ then $\varphi \in \mathrm{BMO}_{\text {prod }}^{d}$.

(vi) $\left\|S_{\varphi}\right\|_{2} \leq C\|\varphi\|_{\mathrm{BMO}_{\text {prod }}^{d}}\|\varphi\|_{2}$.

Here it is the basic result relating the boundedness of $\pi_{b}^{(1,2)}$ and $\Lambda_{S_{b}}$.

Lemma 3.2 Let $b \in \mathcal{H}_{00}$. Then

$$
\pi_{b}^{(1,2)^{*}} \pi_{b}^{(1,2)}=\Lambda_{S_{b}}+D_{b},
$$

where $D_{b}$ is a linear operator on $L^{2}\left(\mathbb{T}^{2}\right)$ with $\left\|D_{b}\right\| \leq C\|b\|_{\mathrm{BMO}_{\mathrm{rect}}^{d}}^{2}$, and $C>0$ is an absolute constant.

Proof. Let $R=I \times J, R^{\prime}=I^{\prime} \times J^{\prime} \in \mathcal{R}$.

First, observe that

$$
\begin{aligned}
& \left\langle\pi_{b}^{(1,2)^{*}} \pi_{b}^{(1,2)} h_{R}, h_{R^{\prime}}\right\rangle \\
& =\left\langle\sum_{I^{\prime \prime} \times J^{\prime \prime} \in \mathcal{D}_{1} \times \mathcal{D}_{2}} h_{I^{\prime \prime} \times J^{\prime \prime}} b_{I^{\prime \prime} \times J^{\prime \prime}} m_{I^{\prime \prime} \times J^{\prime \prime}}\left(h_{R}\right), \sum_{I^{\prime \prime} \times J^{\prime \prime} \in \mathcal{D}_{1} \times \mathcal{D}_{2}} h_{I^{\prime \prime} \times J^{\prime \prime}} b_{I^{\prime \prime} \times J^{\prime \prime}} m_{I^{\prime \prime} \times J^{\prime \prime}}\left(h_{R^{\prime}}\right)\right\rangle \\
& =\sum_{I^{\prime \prime} \times J^{\prime \prime} \in \mathcal{D}_{1} \times \mathcal{D}_{2}, I^{\prime \prime} \subsetneq I, J^{\prime \prime} \subsetneq J}\left|b_{I^{\prime \prime} \times J^{\prime \prime}}\right|^{2} m_{I^{\prime \prime}}\left(h_{I}\right) m_{I^{\prime \prime}}\left(h_{I^{\prime}}\right) m_{J^{\prime \prime}}\left(h_{J}\right) m_{J^{\prime \prime}}\left(h_{J^{\prime}}\right) .
\end{aligned}
$$

We now do a kind of triangular truncation with respect to the indices $I, I^{\prime}, J, J^{\prime}$. 
(i) $I \supsetneq I^{\prime}, J \supsetneq J^{\prime}$.

$$
\begin{aligned}
\left\langle\pi_{S_{b}}^{(1,2)} h_{R},\right. & \left.h_{R^{\prime}}\right\rangle=\left\langle S_{b}, h_{R^{\prime}}\right\rangle m_{R^{\prime}}\left(h_{R}\right) \\
= & \left\langle\sum_{I^{\prime \prime} \times J^{\prime \prime} \in \mathcal{D}_{1} \times \mathcal{D}_{2}} \frac{\chi_{I^{\prime \prime} \times J^{\prime \prime}}}{\left|I^{\prime \prime}\right|\left|J^{\prime \prime}\right|}\left|b_{I^{\prime \prime} \times J^{\prime \prime}}\right|^{2}, h_{R^{\prime}}\right\rangle m_{R^{\prime}}\left(h_{R}\right) \\
= & \sum_{I^{\prime \prime} \times J^{\prime \prime} \in \mathcal{D}_{1} \times \mathcal{D}_{2}}\left|b_{I^{\prime \prime} \times J^{\prime \prime}}\right|^{2} m_{I^{\prime \prime}}\left(h_{I^{\prime}}\right) m_{J^{\prime \prime}}\left(h_{J^{\prime}}\right) m_{I^{\prime}}\left(h_{I}\right) m_{J^{\prime}}\left(h_{J}\right) .
\end{aligned}
$$

This is nonzero only if $I^{\prime} \subsetneq I$ and $J^{\prime} \subsetneq J$. In this case, we get contributions only for $I^{\prime \prime} \subsetneq I^{\prime}$ and $J^{\prime \prime} \subsetneq J^{\prime}$, and the expression agrees with (3.1).

(ii) $I \subsetneq I^{\prime}, J \subsetneq J^{\prime}$. Observe that

$$
\left\langle\Delta_{S_{b}}^{(1,2)} h_{R}, h_{R^{\prime}}\right\rangle=\left\langle h_{R}, \pi_{S_{b}}^{(1,2)} h_{R^{\prime}}\right\rangle=\left\langle\pi_{S_{b}}^{(1,2)} h_{R^{\prime}}, h_{R}\right\rangle .
$$

As shown above, this equals $\left\langle\pi_{b}^{(1,2)^{*}} \pi_{b}^{(1,2)} h_{R^{\prime}}, h_{R}\right\rangle$ if $I^{\prime} \supsetneq I$ and $J^{\prime} \supsetneq J$, and is 0 otherwise.

(iii) $I \supsetneq I^{\prime}, J \subsetneq J^{\prime}$.

$$
\begin{aligned}
\left\langle\pi_{\Delta_{S_{b}}} h_{R}, h_{R^{\prime}}\right\rangle=\left\langle\sum_{I^{\prime \prime} \times J^{\prime \prime} \in \mathcal{D}_{1} \times \mathcal{D}_{2}} S_{I^{\prime \prime} \times J^{\prime \prime}} h_{I^{\prime \prime}} \frac{\chi_{J^{\prime \prime}}}{\left|J^{\prime \prime}\right|} m_{I^{\prime \prime}}\left(h_{R J^{\prime \prime}}\right), h_{R^{\prime}}\right\rangle \\
=\left\langle\sum_{I^{\prime \prime} \in \mathcal{D}_{1}} S_{b I^{\prime \prime} \times J} h_{I^{\prime \prime}} \frac{\chi_{J}}{|J|} m_{I^{\prime \prime}}\left(h_{I}\right), h_{R^{\prime}}\right\rangle \\
=S_{b I^{\prime} \times J^{\prime}} m_{I^{\prime}}\left(h_{J}\right) m_{J}\left(h_{I^{\prime}}\right)=\left\langle S_{b}, h_{I^{\prime} \times J}\right\rangle m_{I^{\prime}}\left(h_{I}\right) m_{J}\left(h_{J^{\prime}}\right) \\
=\left\langle\sum_{I^{\prime \prime} \times J^{\prime \prime} \in \mathcal{D}_{1} \times \mathcal{D}_{2}} \frac{\chi_{I^{\prime \prime} \times J^{\prime \prime}}}{\left|I^{\prime \prime}\right|\left|J^{\prime \prime}\right|}\left|b_{I^{\prime \prime} \times J^{\prime \prime}}\right|^{2}, h_{I^{\prime} \times J}\right\rangle m_{I^{\prime}}\left(h_{I}\right) m_{J}\left(h_{J^{\prime}}\right) \\
=\sum_{I^{\prime \prime} \times J^{\prime \prime} \in \mathcal{D}_{1} \times \mathcal{D}_{2}}\left|b_{I^{\prime \prime} \times J^{\prime \prime}}\right|^{2} m_{I^{\prime \prime}}\left(h_{I^{\prime}}\right) m_{J^{\prime \prime}}\left(h_{J}\right) m_{I^{\prime}}\left(h_{I}\right) m_{J}\left(h_{J^{\prime}}\right) .
\end{aligned}
$$

This is nonzero only for $I^{\prime} \subsetneq I$ and $J^{\prime} \supsetneq J$. In this case, the sum has only contributions for $I^{\prime \prime} \subsetneq I^{\prime}$ and $J^{\prime \prime} \subsetneq J$, and agrees with (3.1).

(iv) $I^{\prime} \supsetneq I$ and $J^{\prime} \subsetneq J$. Note that $\left\langle\Delta_{\pi_{S_{b}}} h_{R}, h_{R^{\prime}}\right\rangle=\left\langle\pi_{\Delta_{S_{b}}} h_{R^{\prime}}, h_{R}\right\rangle$. As shown above, this is only nonzero for $I^{\prime} \supsetneq I$ and $J^{\prime} \subsetneq J$, and agrees with (3.1) in this case. 
(v) $I^{\prime}=I$ or $J=J^{\prime}$. Let $f \in L^{2}\left(\mathbb{T}^{2}\right)$. Then

$$
\begin{aligned}
\sum_{I \in \mathcal{D}_{1}} \sum_{J, J^{\prime} \in \mathcal{D}_{2}}\left\langle\pi_{b}^{(1,2)^{*}} \pi_{b}^{(1,2)} h_{I \times J} f_{I \times J}, h_{I \times J^{\prime}} f_{I \times J^{\prime}}\right\rangle \\
=\sum_{I \in \mathcal{D}_{1}} \frac{1}{|I|} \sum_{I^{\prime \prime} \subsetneq I} \sum_{J^{\prime \prime} \in \mathcal{D}_{2}}\left|b_{I^{\prime \prime} \times J^{\prime \prime}}\right|^{2} f_{I \times J} f_{I \times J^{\prime}} m_{J^{\prime \prime}}\left(h_{J}\right) m_{J^{\prime \prime}}\left(h_{J^{\prime}}\right) \\
=\sum_{I \in \mathcal{D}_{1}}\left\|\pi_{b^{I}} f_{I}\right\|^{2},
\end{aligned}
$$

where for each $I, f_{I}$ stands for the one-variable function

$$
\sum_{J \in \mathcal{D}_{2}} h_{J} f_{I \times J}
$$

and $b^{I}$ for the function

$$
\sum_{J \in \mathcal{D}_{2}} h_{J} \frac{1}{|I|^{1 / 2}}\left(\sum_{I^{\prime \prime} \subsetneq I}\left|b_{I^{\prime \prime} \times J}\right|^{2}\right)^{1 / 2} .
$$

It is easy to see that

$$
\left\|b^{I}\right\|_{\mathrm{BMO}^{d}} \leq\|b\|_{\mathrm{BMO}_{\text {rect }}^{d}} \quad \text { for all } I \in \mathcal{D}_{1} .
$$

Thus the above sum is bounded by $c\|b\|_{\mathrm{BMO}_{\mathrm{rect}}^{d}}^{2}\|f\|^{2}$, where $c$ is an absolute constant.

The same estimate holds for the terms corresponding to $J=J^{\prime}$.

Now we have counted the terms corresponding to $I=I^{\prime}, J=J^{\prime}$ twice and need to estimate them separately. Let $f \in L^{2}\left(\mathbb{T}^{2}\right)$. Then

$$
\begin{aligned}
& \sum_{I \in \mathcal{D}_{1}} \sum_{J \in \mathcal{D}_{2}}\left\langle\pi_{b}^{(1,2)^{*}} \pi_{b}^{(1,2)} h_{I \times J} f_{I \times J}, h_{I \times J} f_{I \times J}\right\rangle \\
& \quad=\sum_{I \in \mathcal{D}_{1}} \sum_{J \in \mathcal{D}_{2}} \frac{1}{|I||J|} \sum_{I^{\prime \prime} \subsetneq I, J^{\prime \prime} \subsetneq J}\left|b_{I^{\prime \prime} \times J^{\prime \prime}}\right|^{2}\left|f_{I \times J}\right|^{2} \leq\|b\|_{\mathrm{BMO}_{\text {rect }}^{d}\|f\|^{2} .}^{2}
\end{aligned}
$$

Defining $D_{b}$ now by

$$
\begin{aligned}
\left\langle D_{b} f, f\right\rangle= & \sum_{I \times J, I^{\prime} \times J^{\prime} \in \mathcal{R}, I^{\prime}=I}\left\langle\pi_{b}^{(1,2)^{*}} \pi_{b}^{(1,2)} h_{I \times J} f_{I \times J}, h_{I^{\prime} \times J^{\prime}} f_{I^{\prime} \times J^{\prime}}\right\rangle+ \\
& +\sum_{I \times J, I^{\prime} \times J^{\prime} \in \mathcal{R}, I^{\prime} \neq I, J^{\prime}=J}\left\langle\pi_{b}^{(1,2)^{*}} \pi_{b}^{(1,2)} h_{I \times J} f_{I \times J}, h_{I^{\prime} \times J^{\prime}} f_{I^{\prime} \times J^{\prime}}\right\rangle,
\end{aligned}
$$

we obtain the statement of the lemma. 
Now we are ready to state the main result of this section.

Theorem 3.3 Let $b \in \mathrm{BMO}_{\text {rect, } 2}^{d}$. Then $b \in \mathrm{BMO}_{\text {prod }}^{d}$ if and only if $S_{b} \in$ $\mathrm{BMO}_{\text {prod }}^{d}$.

Proof. We will first show that there exist $C>0$ such that

$$
\left\|S_{b}\right\|_{\text {prod }} \leq C\|b\|_{\text {prod }}^{2}
$$

Indeed, by Chang's Theorem [Ch], [ChFef2] it is sufficient to show that there exists a constant $C>0$ with

$$
\left\|P_{\Omega} S_{b}\right\|_{2} \leq C\|b\|_{\text {prod }}^{2}|\Omega|^{1 / 2}
$$

for all $\Omega \subseteq \mathbb{T}^{2}$ measurable (see (1.5)). Using Proposition 3.1, we obtain

$$
\left\|P_{\Omega} S_{b}\right\|_{2}=\left\|P_{\Omega} S_{P_{\Omega} b}\right\|_{2} \leq\left\|S_{P_{\Omega} b}\right\|_{2} \leq\left\|P_{\Omega} b\right\|_{\text {prod }}\left\|P_{\Omega} b\right\|_{2} \leq\|b\|_{\text {prod }}^{2}|\Omega|^{1 / 2}
$$

For the converse, assume that $S_{b} \in \mathrm{BMO}_{\text {prod }}^{d}$. Then $\Lambda_{S_{b}}$ is bounded by Theorem 2.8. Now Lemma 3.2 finishes the proof.

Remark. The first implication can also be shown with the John-Nirenberg Theorem for product BMO, which was proved in [ChFef1] (for a dyadic version, see $[\mathrm{T}])$.

The sweep can be understood as a bilinear map. For $f, g \in \mathcal{H}_{00}$, let

$$
S_{f, g}=\sum_{R \in \mathcal{R}} \frac{\chi_{R}}{|R|} f_{r} g_{r}
$$

so $S_{f}=S_{f, \bar{f}}$.

Corollary 3.4 S: $\mathrm{BMO}_{\text {prod }}^{d} \times \mathrm{BMO}_{\text {prod }}^{d} \rightarrow \mathrm{BMO}_{\text {prod }}^{d}$ is bounded.

Proof. The Cauchy-Schwarz inequality gives the pointwise inequality

$$
S_{f, g} \leq\left(S_{f}\right)^{1 / 2}\left(S_{g}\right)^{1 / 2} \quad \text { for } f, g \in \mathcal{H}_{00} .
$$

Let $\Omega \subseteq \mathbb{T}^{2}$ be measurable. Using an adaption of 3.1(iii), we see that

$$
\begin{aligned}
\left\|P_{\Omega} S_{f, g}\right\|_{2}=\left\|P_{\Omega} S_{P_{\Omega} f, P_{\Omega} g}\right\|_{2} & \leq\left\|S_{P_{\Omega} f, P_{\Omega} g}\right\|_{2} \leq\left\|\left(S_{P_{\Omega} f}\right)^{1 / 2}\left(S_{P_{\Omega} g}\right)^{1 / 2}\right\|_{2} \\
& \leq\left\|S_{P_{\Omega} f}\right\|_{2}^{1 / 2}\left\|S_{P_{\Omega} g}\right\|_{2}^{1 / 2} \leq|\Omega|^{1 / 2}\|f\|_{\text {prod }}\|g\|_{\text {prod }}
\end{aligned}
$$

by (3.5). 
Another application of Lemma 3.2 yields the following result.

Theorem 3.5 Let $\|\cdot\|_{*}$ be an positive homogeneous function of degree 1 on $\mathcal{H}_{00}$ such that

(i) There exists $c>0$ such that $\|\cdot\|_{\mathrm{BMO}_{\text {rect }}^{d}} \leq c\|\cdot\|_{*}$

(ii) There exists $k>0$ such that $\left\|S_{\varphi}\right\|_{*} \leq k\|\varphi\|_{*}^{2}$.

Then there exists a constant $\tilde{C}$ such that for all $\varphi \in \mathcal{H}_{00}$,

$$
\|\varphi\|_{\mathrm{BMO}_{\mathrm{prod}}^{d}} \leq \tilde{C}\|\varphi\|_{*}
$$

Proof. Throughout this proof we simply write $\pi_{\varphi}=\pi_{\varphi}^{(1,2)}$. From Lemma 3.2 we have

$$
\pi_{\varphi}^{*} \pi_{\varphi}=\pi_{S_{\varphi}}^{(1,2)}+\pi_{S_{\varphi}}^{(1,2)^{*}}+\pi_{\Delta_{S_{\varphi}}}+\pi_{\Delta_{S_{\varphi}}}{ }^{*}+D_{\varphi}
$$

with $\left\|D_{\varphi}\right\| \leq C\|\varphi\|_{\mathrm{BMO}_{\mathrm{rect}}^{d}}^{2}$.

Let

$$
E_{n}=\operatorname{span}\left\{h_{I \times J}: I \in \mathcal{D}_{1}, J \in \mathcal{D}_{2},|I|,|J| \geq 2^{-n}\right\},
$$

let $P_{n}$ be the orthogonal projection onto $E_{n}$ in $L^{2}\left(\mathbb{T}^{2}\right)$, and let

$$
c(n)=\sup \left\{\left\|\left.\pi_{\varphi}\right|_{E_{n}}\right\|,\|\varphi\|_{*} \leq 1\right\} .
$$

A trivial estimate shows that $c(n)<\infty$ for each $n \in \mathbb{N}$. For $n \in \mathbb{N}$ and $\varepsilon>0$, choose $f_{n} \in E_{n}$ and $\varphi \in \mathcal{H}_{00}$ with $\|\varphi\|_{*}=1,\left\|f_{n}\right\|=1$ and $\left\|\pi_{\varphi} f_{n}\right\| \geq$ $(1-\varepsilon) c(n)$. Then

$$
\begin{aligned}
(1-\varepsilon)^{2} c(n)^{2} \leq & \left\|\pi_{\varphi} f_{n}\right\|^{2}=\left\langle\pi_{\varphi}^{*} \pi_{\varphi} f_{n}, f_{n}\right\rangle \\
= & \left\langle\pi_{S_{\varphi}}^{(1,2)} f_{n}, f_{n}\right\rangle+\left\langle\pi_{S_{\varphi}}^{(1,2)^{*}} f_{n}, f_{n}\right\rangle+ \\
& \quad+\left\langle\pi_{\Delta_{S_{\varphi}}} f_{n}, f_{n}\right\rangle+\left\langle\pi_{\Delta_{S_{\varphi}}}^{*} f_{n}, f_{n}\right\rangle+\left\langle D_{\varphi} f_{n}, f_{n}\right\rangle .
\end{aligned}
$$

By definition of $c(n)$, the first two terms can be estimated by

$$
c(n)\left\|S_{\varphi}\right\|_{*} \leq c(n) k .
$$

For the next two terms, we have to remark that that

$$
\left\langle\pi_{\Delta_{S_{\varphi}}} f_{n}, f_{n}\right\rangle=\left\langle\pi_{\Delta_{P_{n} S_{\varphi}}} f_{n}, f_{n}\right\rangle \leq\left\|\pi_{\Delta_{P_{n} S_{\varphi}}}\right\| \leq \tilde{c}\left\|\pi_{P_{n} S_{\varphi}}^{(1,2)}\right\| \leq \tilde{c}\left\|\left.\pi_{S_{\varphi}}^{(1,2)}\right|_{E_{n}}\right\|
$$

Here, we use as in the proof of Thm 2.8 that there exists a constant $\tilde{c}$ such that $\left\|\pi_{\Delta_{b}}\right\| \leq \tilde{c}\left\|\pi_{b}^{(1,2)}\right\|$ for all $b \in \mathcal{H}_{00}$ (see [PS], Thm 7.7.2).

The last term is easily controlled by

$$
\left\langle D_{\varphi} f_{n}, f_{n}\right\rangle \leq C\|\varphi\|_{\mathrm{BMO}_{\mathrm{rect}}^{d}}^{2} .
$$


Altogether, we obtain that

$$
\left\|\left.\pi_{\varphi}^{(1,2)}\right|_{E_{n}}\right\|^{2} \leq 4 \tilde{c}\left\|\left.\pi_{S_{\varphi}}^{(1,2)}\right|_{E_{n}}\right\|+C\|\varphi\|_{\mathrm{BMO}_{\mathrm{rect}}^{d}}^{2} .
$$

With

$$
\|\varphi\|_{\mathrm{BMO}_{\text {rect }}^{d}} \leq c\|\varphi\|_{*}
$$

and

$$
\left\|\left.\pi_{S_{\varphi}}^{(1,2)}\right|_{E_{n}}\right\| \leq c(n)\left\|S_{\varphi}\right\|_{*} \leq k c(n)\|\varphi\|_{*}^{2},
$$

it follows that

$$
(1-\varepsilon)^{2} c(n)^{2} \leq 4 \tilde{c} k c(n)+c^{2} C
$$

Thus

$$
c(n) \leq \sqrt{4 \tilde{c}^{2} k^{2}+C c^{2}}+2 \tilde{c} k .
$$

With

$$
\tilde{C}=\sqrt{4 \tilde{c}^{2} k^{2}+C c^{2}}+2 \tilde{c} k,
$$

it follows that $\left\|\pi_{\varphi}\right\| \leq \tilde{C}\|\varphi\|_{*}$.

We can now characterize $\mathrm{BMO}_{\text {prod }}^{d}$ in terms of the $\mathrm{BMO}_{\text {rect, } 2 \text {-norm. }}^{d}$

Theorem 3.6 Let $\varphi \in \mathrm{BMO}_{\text {rect }}^{d}\left(\mathbb{T}^{2}\right)$. Then $\varphi \in \mathrm{BMO}_{\text {prod }}^{d}$ if and only if $\left(\left\|S_{\varphi}^{(n)}\right\|_{\mathrm{BMO}_{\text {rect }}^{d}}^{1 / 2^{n}}\right)_{n \in \mathbb{N}}$ is bounded, where $S_{\varphi}^{(n)}$ is the $n$-fold sweep of $\varphi$, defined recursively by $S_{\varphi}^{(n)}=S_{S_{\varphi}}^{(n-1)}$.

Proof. By Theorem 3.3, we have for each $n \in \mathbb{N}$

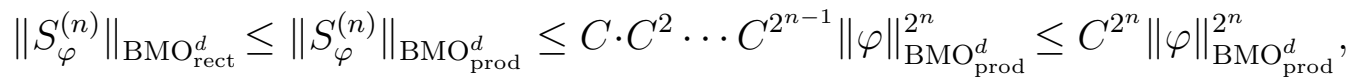
and consequently

$$
\left\|S_{\varphi}^{(n)}\right\|_{\mathrm{BMO}_{\text {rect }}^{d}}^{1 / 2^{n}} \leq C\|\varphi\|_{\mathrm{BMO}_{\mathrm{prod}}^{d}} .
$$

Conversely, the map

$$
\varphi \mapsto \sup _{n \in \mathbb{N}}\left\|S_{\varphi}^{(n)}\right\|_{\mathrm{BMO}_{\text {rect }}^{1 / 2^{n}}}^{d}
$$

clearly defines a positive homogeneous function on $\mathcal{H}_{00}$ with satisfies conditions in Theorem 3.5.

Another consequence of Theorem 3.5 is

Corollary 3.7 S does not map $\mathrm{BMO}_{\text {rect }}^{d} \times \mathrm{BMO}_{\text {rect }}^{d}$ boundedly into $\mathrm{BMO}_{\text {rect }}^{d}$.

Proof. We know that the $\|\cdot\|_{\text {prod }}$ norm cannot be controlled by the $\|\cdot\|_{\text {rect }}$ norm. So Condition (ii) in Theorem 3.5 cannot hold, and in particular $S$ does not map $\mathrm{BMO}_{\text {rect }}^{d} \times \mathrm{BMO}_{\text {rect }}^{d}$ boundedly into $\mathrm{BMO}_{\text {rect }}^{d}$. 


\section{The scale $\mathrm{BMO}_{\text {rect, } p}^{d}$}

Recall that for $1 \leq p<\infty$, a function $\varphi \in L^{2}\left(\mathbb{T}^{2}\right)$ is said to belong to $\mathrm{BMO}_{\text {rect }, p}^{d}$ if

$$
\|\varphi\|_{\text {rect }, p}=\sup _{R \in \mathcal{R}} \frac{1}{|R|^{1 / p}}\left\|P_{R} \varphi\right\|_{p}<\infty .
$$

Note that $\mathrm{BMO}_{\text {rect, }, p_{2}}^{d} \subseteq \mathrm{BMO}_{\text {rect, } p_{1}}^{d}$ for $p_{1} \leq p_{2}$.

The reader should also be aware that functions in $\mathrm{BMO}_{\text {rect, }, p}^{d}$ are actually in $L^{p}\left(\mathbb{T}^{2}\right)$, due to the identities $m_{I}(f)=m_{I}\left(P_{I \times \mathbb{T}} f\right)$ and $m_{J}(f)=$ $m_{J}\left(P_{\mathbb{T} \times J} f\right)$.

The following proposition characterizes the behaviour of the $\mathrm{BMO}_{\text {rect }, p}^{d}$ norms under the sweep.

Proposition 4.1 Let $p>\frac{1}{2}$ and let $C_{p}=\|\mathcal{S}\|_{L^{2 p} \rightarrow L^{2 p}}$. Then

$$
\left\|S_{\varphi}\right\|_{\text {rect }, p} \leq 4 C_{p}^{2}\|\varphi\|_{\text {rect }, 2 p}^{2} .
$$

Proof. Since $P_{R}\left(S_{\varphi}\right)=P_{R}\left(S_{P_{R} \varphi}\right)$ and $\left\|P_{R}(g)\right\|_{p} \leq 4\|g\|_{p}$, we obtain

$$
\left\|S_{\varphi}\right\|_{\text {rect }, p} \leq 4 \sup _{R \in \mathcal{R}} \frac{1}{|R|^{1 / p}}\left\|S_{P_{R}(\varphi)}\right\|_{p} \leq 4 C_{p}^{2} \sup _{R \in \mathcal{R}} \frac{1}{|R|^{1 / p}}\left\|P_{R}(\varphi)\right\|_{2 p}^{2} .
$$

This gives the result.

It is known that $\mathrm{BMO}_{\text {prod }}^{d} \subsetneq \mathrm{BMO}_{\text {rect,2. Indeed, this is basically the content }}^{d}$ of Carleson's original counterexample [C] (for the continuous case, see [Fef]). As pointed out in [Fef], the example in [C] implies that $\mathrm{BMO}_{\text {rect, } 2}^{d} \not L^{4}\left(\mathbb{T}^{2}\right)$.

We shall improve this by showing that actually $\mathrm{BMO}_{\text {prod }}^{d} \subsetneq \mathrm{BMO}_{\text {rect }, p}^{d}$ for all $p$. We will show that for any $p_{2}>p_{1} \geq 1, \mathrm{BMO}_{\text {rect, } p_{1}}^{d} \nsubseteq L^{p_{2}}\left(\mathbb{T}^{2}\right)$ and therefore in particular $\mathrm{BMO}_{\text {rect, } p_{1}}^{d} \nsubseteq \mathrm{BMO}_{\text {rect, } p_{2}}^{d}$. For the case $p_{1}=1, p_{2}=2$, this answers a question posed in [FS].

As a corollary, we show that

$$
\mathrm{BMO}_{\text {prod }}^{d} \subsetneq \bigcap_{p \geq 1} \mathrm{BMO}_{\text {rect }, p}^{d}
$$

Theorem 4.2 Let $p \geq 2$. Then $\mathrm{BMO}_{\text {prod }}^{d} \subseteq \mathrm{BMO}_{\text {rect }, p}^{d}$.

Moreover

$$
\|\varphi\|_{\text {rect }, p} \leq C\|\varphi\|_{\text {prod }}^{1-2 / p}\|\varphi\|_{\text {rect }, 2}^{2 / p}
$$


Proof. Let us first show that $\mathrm{BMO}_{\text {prod }}^{d} \subseteq L^{p}\left(\mathbb{T}^{2}\right)$ and

$$
\|\varphi\|_{p} \leq C\|\varphi\|_{\text {prod }}^{1-2 / p}\|\varphi\|_{2}^{2 / p} \text {. }
$$

For $p=2^{k}, k \in \mathbb{N}$, we shall prove (4.2) by induction.

It is obvious for $k=1$. For $k=2$ we have

$$
\|\varphi\|_{4}^{2}=\left\|S_{\varphi}\right\|_{2}=\left\|\Delta_{\varphi}^{(1,2)}(\varphi)\right\|_{2} \leq C\|\varphi\|_{\text {prod }}\|\varphi\|_{2} .
$$

Assume it holds for $p_{k}=2^{k}$.

$$
\|\varphi\|_{p_{k+1}}^{2}=\left\|S_{\varphi}\right\|_{p_{k}} \leq C\left\|S_{\varphi}\right\|_{\text {prod }}^{1-2 / p_{k}}\left\|S_{\varphi}\right\|_{2}^{2 / p_{k}}
$$

Now from (3.4) and (4.3) we obtain

$$
\|\varphi\|_{p_{k+1}} \leq C\|\varphi\|_{\text {prod }}^{1-2 / p_{k}}\|\varphi\|_{4}^{2 / p_{k}} \leq C\|\varphi\|_{\text {prod }}^{1-2 / p_{k+1}}\|\varphi\|_{2}^{2 / p_{k+1}} .
$$

Now the general case follows by interpolation.

Given $p>2$ and $p \neq 2^{k}$ for any $k \in \mathbb{N}$, find $m \in \mathbb{N}$ such that $2^{m-1}<p<2^{m}$. Write

$$
\frac{1}{p}=\frac{1-\theta_{m}}{2^{m-1}}+\frac{\theta_{m}}{2^{m}}
$$

Now apply the previous case combined with

$$
\|\varphi\|_{p} \leq\|\varphi\|_{2^{m-1}}^{1-\theta_{m}}\|\varphi\|_{2^{m}}^{\theta_{m}}
$$

Let us use (4.2) to obtain the desired estimate for the $\mathrm{BMO}_{\text {rect, }, p}^{d}$-norm. Given $R \in \mathcal{R}$ we have

$$
\left\|P_{R} \varphi\right\|_{p} \leq C\left\|P_{R} \varphi\right\|_{\text {prod }}^{1-2 / p}\left\|P_{R} \varphi\right\|_{2}^{2 / p} \leq C\|\varphi\|_{\text {prod }}^{1-2 / p}\|\varphi\|_{\text {rect }, 2}^{2 / p}|R|^{1 / p} .
$$

This finishes the proof.

Proposition 4.3 Let $2<p$. There exists $\phi \in \mathrm{BMO}_{\text {rect, } 2}^{d} \backslash L^{p}\left(\mathbb{T}^{2}\right)$.

In particular $\mathrm{BMO}_{\text {prod }}^{d} \subseteq \mathrm{BMO}_{\text {rect }, p}^{d} \subsetneq \mathrm{BMO}_{\text {rect }, 2}^{d}$.

Proof. We shall find a sequence $\varphi_{N}$ such that $\sup _{N}\left\|\varphi_{N}\right\|_{\text {rect, } 2}<\infty$ but $\sup _{N}\left\|\varphi_{N}\right\|_{p}=\infty$. A standard argument then gives the existence of $\phi$.

From Carleson's construction [C] we know that for each $N \in \mathbb{N}$ there exists a collection of dyadic rectangles $\Phi_{N}$ such that

$$
\begin{aligned}
\sum_{R \in \Phi_{N}}|R| & =1 \\
\left|\bigcup_{R \in \Phi_{N}} R\right| & <\frac{1}{N} \\
\sum_{R \in \Phi_{N}, R \subseteq R^{\prime}}|R| & \leq C\left|R^{\prime}\right|, \quad R^{\prime} \in \mathcal{R}
\end{aligned}
$$


where $C$ is a constant independent of $N$.

Defining

$$
\varphi_{N}=\sum_{R \in \Phi_{N}}|R|^{1 / 2} h_{R}
$$

we have that

$$
\left\|\varphi_{N}\right\|_{2}=1, \quad\left\|\varphi_{N}\right\|_{\text {rect }, 2} \leq C
$$

but, since $\operatorname{supp}\left(\varphi_{N}\right) \subset \cup_{R \in \Phi_{N}} R$,

$$
\left\|\varphi_{N}\right\|_{p} \geq\left|\bigcup_{R \in \Phi_{N}} R\right|^{\frac{1}{p}-\frac{1}{2}} \geq N^{\frac{1}{2}-\frac{1}{p}}
$$

We now can answer in the negative the above mentioned question of C. Sadosky and S. Ferguson posed in [FS].

Corollary 4.4 There exists $\phi \in \mathrm{BMO}_{\text {rect, } 1}^{d} \backslash \bigcup_{p>1} L^{p}\left(\mathbb{T}^{2}\right)$. In particular, for each $p>1, \mathrm{BMO}_{\text {rect }, p}^{d} \subsetneq \mathrm{BMO}_{\text {rect, } 1}^{d}$, and the norms $\|\cdot\|_{\text {rect, } 1}$ and $\|\cdot\|_{\text {rect }, p}$ are not equivalent.

Proof. We use the sequence of functions $\left(\varphi_{n}\right)_{n \in \mathbb{N}}$ with

$$
\left\|\varphi_{n}\right\|_{\text {rect }, 2} \leq C \text { and }\left\|\varphi_{n}\right\|_{p} \geq n^{1 / 2-1 / p}
$$

for each $n \in \mathbb{N}, p>2$ from Proposition 4.3.

Define

$$
\phi=\sum_{n=1}^{\infty} \frac{1}{n^{2}} S_{\varphi_{2^{n}}} .
$$

Then $\phi \in \mathrm{BMO}_{\text {rect, } 1}$ by Proposition 4.1 , but $\left\|S_{\varphi_{2^{n}}}\right\|_{p} \approx\left\|\varphi_{2^{n}}\right\|_{2 p}^{2} \geq 2^{n(1-1 / p)}$ for each $n \in \mathbb{N}, p>1$ and consequently $\phi \notin \cup_{p>1} L^{p}\left(\mathbb{T}^{2}\right)$.

To differentiate the spaces $\mathrm{BMO}_{\text {rect, } p}^{d}$ and $\mathrm{BMO}_{\text {prod }}^{d}$ we shall introduce the following coefficients.

Definition 4.5 Let $E_{n}=\operatorname{span}\left\{h_{I \times J}: I \in \mathcal{D}_{1}, J \in \mathcal{D}_{2},|I|,|J| \geq 2^{-n}\right\}$ and let $P_{n}$ be the orthogonal projection onto $E_{n}$ in $L^{2}\left(\mathbb{T}^{2}\right)$.

For each $q \geq 1$ and each $n \in \mathbb{N}$

$$
c(n, q)=\sup \left\{\left\|\pi_{\varphi}\right\|: \varphi \in E_{n},\|\varphi\|_{\text {rect }, q} \leq 1\right\},
$$

and for $p \geq q$,

$$
a(n, p, q)=\sup \left\{\|\varphi\|_{\text {rect }, p}: \varphi \in E_{n},\|\varphi\|_{\text {rect }, q} \leq 1\right\},
$$


We first analyse the behaviour of these constants. Of course we have

$$
\begin{aligned}
c\left(n, p_{2}\right) & \leq c\left(n, p_{1}\right), \quad p_{1} \leq p_{2} \\
a\left(n, p, q_{1}\right) & \leq a\left(n, p, q_{2}\right), \quad q_{1} \leq q_{2} \\
a\left(n, p_{2}, q\right) & \leq a\left(n, p_{1}, q\right), \quad p_{1} \leq p_{2} .
\end{aligned}
$$

If $p \geq q$, clearly

$$
c(n, q) \leq a(n, p, q) c(n, p) .
$$

Let us now extend Therorem 4.2.

Theorem 4.6 Let $p>q \geq 2$ and $\varphi \in \mathrm{BMO}_{\text {prod. }}^{d}$. If $q \leq 2^{k} \leq p$ for some $k \in \mathbb{N}$ then

$$
\|\varphi\|_{\text {rect }, p} \leq C_{p, q}\|\varphi\|_{\text {prod }}^{1-q / p}\|\varphi\|_{\text {rect }, q}^{q / p} .
$$

In particular, for $p>q_{1} \geq q_{2} \geq 2$ we have

$$
a\left(n, p, q_{2}\right) \leq C_{p} c\left(n, q_{2}\right)^{1-q_{1} / p} a\left(n, q_{1}, q_{2}\right)^{q_{1} / p}
$$

Proof. We shall see first that

$$
\|\varphi\|_{p} \leq C_{p, q}\|\varphi\|_{\text {prod }}^{1-\theta}\|\varphi\|_{q}^{\theta}
$$

for the above values of $\theta=q / p$. We do this in several steps.

First suppose that $q=2^{n}$ for some $n \in \mathbb{N}$. Theorem 4.2 gives the case $n=1$. Assume that the result is true for $n \geq 2$, and let us consider the case $q=2^{n+1}$.

Let $p>2^{n+1}$. Applying the induction assumption to $S_{\varphi}$ for $p / 2$, we get

$$
\begin{aligned}
\|\varphi\|_{p}^{2} & \approx\left\|S_{\varphi}\right\|_{p / 2} \\
& \leq C\left\|S_{\varphi}\right\|_{\text {prod }}^{1-2^{n+1} / p}\left\|S_{\varphi}\right\|_{2^{n}}^{2^{n+1} / p} \\
& \leq C\|\varphi\|_{\operatorname{prod}}^{2\left(1-2^{n+1} / p\right)}\|\varphi\|_{2^{n+1}}^{2^{n+2} / p} .
\end{aligned}
$$

This shows that

$$
\|\varphi\|_{p} \leq C\|\varphi\|_{\text {prod }}^{1-2^{n+1} / p}\|\varphi\|_{2^{n+1}}^{2^{n+1} / p} .
$$

Let us now proceed to the general case. We may assume that $q<2^{k} \leq p$ for some $k \in N$. We can apply the previous case for $n=k$ together with interpolation. Writing

$$
\frac{1}{2^{k}}=\frac{1-\alpha}{q}+\frac{\alpha}{p}
$$


we obtain

$$
\begin{aligned}
\|\varphi\|_{p} & \leq C\|\varphi\|_{\text {prod }}^{1-2^{k} / p}\|\varphi\|_{2^{k}}^{2^{k} / p} \\
& \leq C\|\varphi\|_{\text {prod }}^{1-2^{k} / p}\left(\|\varphi\|_{q}^{1-\alpha}\|\varphi\|_{p}^{\alpha}\right)^{2^{k} / p}
\end{aligned}
$$

Consequently

$$
\|\varphi\|_{p}^{1-\alpha 2^{k} / p} \leq C\|\varphi\|_{\operatorname{prod}}^{1-2^{k} / p}\|\varphi\|_{q}^{(1-\alpha) 2^{k} / p}
$$

Note that

$$
(1-\alpha) \frac{2^{k}}{q}=1-\alpha \frac{2^{k}}{p}
$$

Hence we get with $\theta=q / p$ that

$$
\|\varphi\|_{p} \leq C\|\varphi\|_{\text {prod }}^{1-\theta}\|\varphi\|_{q}^{\theta} .
$$

To finish the proof, note that for each $R \in \mathcal{R}$,

$$
\begin{aligned}
\left\|P_{R} \varphi\right\|_{p} & \leq C_{p, q}\left\|P_{R} \varphi\right\|_{\text {prod }}^{1-q / p}\left\|P_{R} \varphi\right\|_{q}^{q / p} \\
& \leq C_{p, q}\|\varphi\|_{\text {prod }}^{1-q / p}\|\varphi\|_{\text {rect }, q}^{q / p}|R|^{1 / p}
\end{aligned}
$$

Let us now establish a further connection between the constants introduced in 4.5.

Theorem 4.7 There exist $K_{1}>0$ and $K_{2}>0$ such that for all $n \in \mathbb{N}$ and $p \geq 1$

$$
c^{2}(n, 2 p) \leq K_{1} C_{p}^{2} c(n, p)+K_{2},
$$

where $C_{p}=\|\mathcal{S}\|_{L^{2 p} \rightarrow L^{2 p}}$.

Proof. Write

$$
\pi_{\varphi}^{*} \pi_{\varphi}=\Lambda_{S_{\varphi}}+D_{\varphi}
$$

with $\left\|D_{\varphi}\right\| \leq C\|\varphi\|_{\mathrm{BMO}_{\text {rect }}^{d}}^{2}$ as above.

For $n \in \mathbb{N}, p \geq 1$ and $\varepsilon>0$, choose $f_{n} \in L^{2}\left(\mathbb{T}^{2}\right)$ and $\varphi \in E_{n}$ with $\|\varphi\|_{\text {rect }, 2 p}=1,\left\|f_{n}\right\|_{2}=1$ and $\left\|\pi_{\varphi} f_{n}\right\|_{2} \geq(1-\varepsilon) c(n, 2 p)$. Then

$$
(1-\varepsilon)^{2} c(n, 2 p)^{2} \leq\left\|\pi_{\varphi} f_{n}\right\|_{2}^{2}=\left\langle\pi_{\varphi}^{*} \pi_{\varphi} f_{n}, f_{n}\right\rangle=\left\langle\Lambda_{S_{\varphi}} f_{n}, f_{n}\right\rangle+\left\langle D_{\varphi} f_{n}, f_{n}\right\rangle .
$$

Therefore, we obtain that

$$
(1-\varepsilon)^{2} c(n, 2 p)^{2} \leq\left\|\Lambda_{S_{\varphi}}\right\|+C\|\varphi\|_{\mathrm{BMO}_{\mathrm{rect}}^{d}}^{2}
$$


Since $\|\varphi\|_{\text {rect }, 2 p}=1$, Proposition 4.1 implies $\left\|S_{\varphi}\right\|_{\text {rect }, p} \leq 4 C_{p}^{2}$. Therefore, since $\|\varphi\|_{\text {rect }, 2} \leq\|\varphi\|_{\text {rect }, 2 p}$, it follows that

$$
(1-\varepsilon)^{2} c(n, 2 p)^{2} \leq 4 C_{p}^{2} c(n, p)+C .
$$

Using (4.10) we get the second part.

Corollary 4.8 Let $p \geq 1$. Then $\mathrm{BMO}_{\text {prod }}^{d} \subsetneq \mathrm{BMO}_{\text {rect }, p}^{d}$.

Proof. Observe first that Proposition 4.3 implies that

$$
\lim _{n \rightarrow \infty} c(n, 2)=\infty \text {. }
$$

This shows that $\mathrm{BMO}_{\text {prod }}^{d} \subsetneq \mathrm{BMO}_{\text {rect }, p}^{d}$ for any $1 \leq p \leq 2$.

On the other hand, the estimates (4.10) and (4.12) in the case $q=q_{1}=q_{2}$ imply that if $p>q \geq 2$ with $q \leq 2^{k} \leq p$ for some $k$,

$$
c^{q / p}(n, q) \leq C c(n, p),
$$

where $C$ is independent of $n$.

Hence $\mathrm{BMO}_{\text {prod }}^{d}=\mathrm{BMO}_{\text {rect, } p}^{d}$ for some $p>2$ would imply $\sup _{n} c(n, p)<\infty$ and therefore $\sup _{n} c(n, 2)<\infty$, contradicting (4.16).

The particular case $p=4$ means that a question left open in [PS] can be answered in the negative. There, it was asked whether the condition

$$
\begin{aligned}
& \left\|\left(\pi_{b}^{(1,2)}\right)^{*} \pi_{b}^{(1,2)} h_{I^{\prime}} f\right\|^{2}=\frac{1}{\left|I^{\prime}\right|}\left\|\sum_{I \times J \in \mathcal{D}_{1} \times \mathcal{D}_{2}, I \subsetneq I^{\prime}} \frac{\chi_{I \times J}}{|I||J|}\left|b_{I J}\right|^{2} m_{J} f\right\|_{L^{2}\left(\mathbb{T}^{2}\right)}^{2} \\
& \leq C\|f\|_{L^{2}(\mathbb{T})} \quad\left(f \in L^{2}(\mathbb{T}), I^{\prime} \in \mathcal{D}_{1}\right)
\end{aligned}
$$

$((27)$ and $(28)$ in $[\mathrm{PS}])$ already implies that $b \in \mathrm{BMO}_{\text {prod }}^{d}$. Note that $f$ here denotes a function in the second variable. We know from Prop 4.1 that $b \in \mathrm{BMO}_{\text {rect }, 4}$ implies $S_{b} \in \mathrm{BMO}_{\text {rect }}^{d}$. By Lemma 3.2,

$$
\left\|\left(\pi_{b}^{(1,2)}\right)^{*} \pi_{b}^{(1,2)} h_{I^{\prime}} f\right\|=\left\|\left(\Lambda_{S_{b}}+D_{b}\right) h_{I^{\prime}} f\right\|,
$$

where $D_{b}$ is bounded on $L^{2}\left(\mathbb{T}^{2}\right)$ and $\Lambda_{S_{b}}$ maps $L^{2}(\mathbb{T}) \hat{\otimes} L^{2}(\mathbb{T})$ boundedly into $L^{2}\left(\mathbb{T}^{2}\right)$ by Prop 2.11. Thus $b \in \mathrm{BMO}_{\text {rect, } 4}$ implies (4.18). This condition is therefore not sufficient for $b \in \mathrm{BMO}_{\text {prod }}^{d}$.

As pointed out in [PS], this has also consequences for the study of operator-valued Carleson measures, in the sense that a certain vector BMO condition of the sweep of an operator-valued measure does not imply boundedness of the corresponding vector Carleson embedding.

We can further show that even the intersection of all $\mathrm{BMO}_{\text {rect,p }}^{d}$ spaces is still bigger than $\mathrm{BMO}_{\text {prod }}^{d}$. 


\section{Corollary 4.9}

$$
\mathrm{BMO}_{\text {prod }}^{d} \subsetneq \bigcap_{p \geq 1} \mathrm{BMO}_{\text {rect }, p}^{d}
$$

Proof. Obviously

$$
\bigcap_{p \geq 1} \mathrm{BMO}_{\mathrm{rect}, p}^{d}=\bigcap_{p \in \mathbb{N}} \mathrm{BMO}_{\mathrm{rect}, p}^{d}
$$

With the locally convex topology defined by the increasing sequence of seminorms $\left(\|\cdot\|_{\text {rect, } p}\right)_{p \in \mathbb{N}}$, the latter is a metrizable locally convex linear space. Since each of the $\mathrm{BMO}_{\text {rect, } p}^{d}$ is complete in $\|\cdot\|_{\text {rect }, p}, \cap_{p \in \mathbb{N}} \mathrm{BMO}_{\text {rect, } p}^{d}$ is complete in this topology and therefore a Fréchet space. We know from Theorem 4.2 that

$$
\mathrm{BMO}_{\text {prod }}^{d} \subseteq \bigcap_{p \geq 1} \mathrm{BMO}_{\text {rect }, p}^{d},
$$

and that the embedding is continuous with respect to the norm topology on $\mathrm{BMO}_{\text {prod }}^{d}$ and the locally convex topology on $\bigcap_{p \geq 1} \mathrm{BMO}_{\text {rect }, p}$. Let us assume towards a contradiction that the embedding is surjective. Then the open mapping theorem implies that the locally convex topology on $\bigcap_{p \geq 1} \mathrm{BMO}_{\text {rect, } p}^{d}$ is normable with $\|\cdot\|_{\text {prod }}$ and therefore contains a nonempty open neighbourhood of 0 which is bounded with respect to $\|\cdot\|_{\text {prod }}$. Since the family $\left(\|\cdot\|_{\text {rect, } p}\right)_{p \in \mathbb{N}}$ is increasing, this means that there exists $p \in \mathbb{N}$ and $\varepsilon>0$ such that $\|b\|_{\text {prod }}<1$ whenever $\|b\|_{\text {rect }, p}<\varepsilon$, in contradiction to Corollary 4.8.

We will now separate the $\mathrm{BMO}_{\text {rect, } p}^{d}$ spaces. Note that for Corollary 4.4 means that

$$
\lim _{n \rightarrow \infty} a(n, p, 1)=\infty
$$

for all $p>1$.

Let us see that this holds in general.

Corollary 4.10 Let $p>q \geq 1$. Then $\mathrm{BMO}_{\text {rect }, p}^{d} \subsetneq \mathrm{BMO}_{\text {rect, }, q}^{d}$.

Proof. We have to show that

$$
\lim _{n \rightarrow \infty} a(n, p, q)=\infty
$$

It suffices to prove

$$
\lim _{n \rightarrow \infty} a(n, q+\varepsilon, q)=\infty
$$

for sufficiently small $\varepsilon$. For fixed $q>1$, choose $\varepsilon>0$ and $k \in \mathbb{N}$ such that $q<q+\varepsilon<2^{k} \leq 2 q$. 
Using Theorem 4.7, (4.10) and (4.17), we obtain constants $C_{1}, C_{2}$ and $C_{3}$ independent of $n$ such that

$$
\begin{aligned}
c^{2}(n, 2 q) & \leq C_{1} c(n, q) \leq C_{1} C_{2} a(n, q+\varepsilon, q) c(n, q+\varepsilon) \\
& \leq C_{1} C_{2} C_{3} a(n, q+\varepsilon, q) c(n, 2 q)^{\frac{2 p}{p+\varepsilon}} .
\end{aligned}
$$

This shows that

$$
c(n, 2 q)^{\frac{2 \varepsilon}{p+\varepsilon}} \leq C a(n, q+\varepsilon, q)
$$

where $C$ is independent of $n$. Now the result follows from Corollary 4.8.

Acknowledgement. We thank C. Sadosky for fruitful discussions.

\section{References}

[Be] Bernard, A.: Espaces $H^{1}$ de martingales à deux indices. Dualité avec les martingales de type "BMO" (French). Bull. Sci. Math. (2) 103 (1979), no. 3, 297-303.

[C] Carleson, L.: A counterexample for measures bounded on $H^{p}$ for the bi-disc. Mittag-Leffler Report No. 7, 1974.

[Ch] Chang, S.-Y. A.: Carleson measure on the bi-disc. Ann. of Math. (2) 109 (1979), no. 3, 613-620.

[ChFef1] Chang, S.-Y.A. And Fefferman, R.: A continuous version of duality of $H^{1}$ with BMO on the bidisc. Ann. of Math. (2) 112 (1980), no. $1,179-201$.

[ChFef2] Chang, S.-Y.A. And Fefferman, R.: Some recent developments in Fourier analysis and $H^{p}$ theory on product domains. Bull. Amer. Math. Soc. (N. S.) 12 (1985), 1-43.

[Fef] Fefferman, R.: Bounded mean oscillation on the polydisc. Ann. of Math. (2) 110 (1979), 395-406.

[FS] Ferguson, S. And Sadosky, C.: Characterizations of bounded mean oscillation on the polydisk in terms of Hankel operators and Carleson measures. J. Anal. Math. 81 (2000), 239-267.

[GPTV] Gillespie, T.A., Pott, S., Treil, S. And Volberg, A.: Logarithmic growth for matrix martingale transforms. J. London Math. Soc. (3) 64 (2001), 624-636.

[LF] Lacey, M. And Ferguson, S.: A characterization of product BMO by commutators. Acta Math. 189 (2002), no. 2, 143-160.

[Per] Pereyra, C. M.: Lecture notes on dyadic harmonic analysis. In Second Summer School in Analysis and Mathematical Physics (Cuernavaca, 2000), 1-60. Contemp. Math. 289. Amer. Math. Soc., Providence, 2001. 
[PS] Pott, S. And Sadosky, C.: Bounded mean oscillation on the bidisk and Operator BMO. J. Funct. Anal. 189 (2002), 475-495.

[T] TAO, T.: Dyadic product $H^{1}$, BMO and Carleson's Counterexample. Short stories, http://www.math.ucla.edu/ tao/

Recibido: 7 de febrero de 2003

Revisado: 2 de octubre de 2003

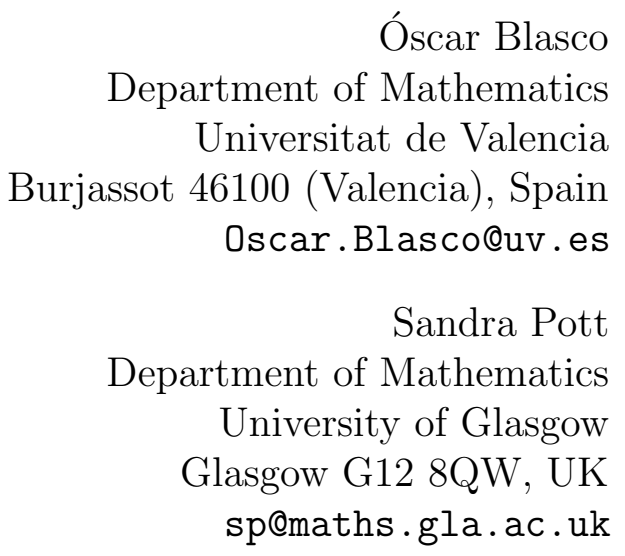

The first author gratefully acknowledges support by the Spanish grants PR2001-0134, BFM2002-04013 and by the LMS. The second author acknowledges support by EPSRC and by the Nuffield Foundation. 